\title{
THE STRUCTURE OF SELF-SIMILAR STABLE MIXED MOVING AVERAGES ${ }^{1}$
}

\author{
By Vladas Pipiras and Murad S. TAQQU \\ Boston University
}

Let $\alpha \in(1,2)$ and $X_{\alpha}$ be a symmetric $\alpha$-stable $(S \alpha S)$ process with stationary increments given by the mixed moving average

$$
X_{\alpha}(t)=\int_{X} \int_{\mathbb{R}}(G(x, t+u)-G(x, u)) M_{\alpha}(d x, d u), \quad t \in \mathbb{R},
$$

where $(X, \mathcal{X}, \mu)$ is a standard Lebesgue space, $G: X \times \mathbb{R} \mapsto \mathbb{R}$ is some measurable function and $M_{\alpha}$ is a $S \alpha S$ random measure on $X \times \mathbb{R}$ with the control measure $m(d x, d u)=\mu(d x) d u$. Assume, in addition, that $X_{\alpha}$ is self-similar with exponent $H \in(0,1)$. In this work, we obtain a unique in distribution decomposition of a process $X_{\alpha}$ into three independent processes

$$
X_{\alpha} \stackrel{d}{=} X_{\alpha}^{(1)}+X_{\alpha}^{(2)}+X_{\alpha}^{(3)} \text {. }
$$

We characterize $X_{\alpha}^{(1)}$ and $X_{\alpha}^{(2)}$ and provide examples of $X_{\alpha}^{(3)}$.

The first process $X_{\alpha}^{(1)}$ can be represented as

$$
\int_{Y} \int_{\mathbb{R}} \int_{\mathbb{R}} e^{-\kappa s}\left(F\left(y, e^{s}(t+u)\right)-F\left(y, e^{s} u\right)\right) M_{\alpha}(d y, d s, d u),
$$

where $\kappa=H-1 / \alpha,(Y, \mathcal{y}, v)$ is a standard Lebesgue space and $M_{\alpha}$ is a $S \alpha S$ random measure on $Y \times \mathbb{R} \times \mathbb{R}$ with the control measure $m(d y, d s, d u)=$ $v(d y) d s d u$. Particular cases include the limit of renewal reward processes, the so-called "random wavelet expansion" and Takenaka process. The second process $X_{\alpha}^{(2)}$ has the representation

$$
\begin{array}{lr}
\int_{Z} \int_{\mathbb{R}}\left(G_{1}(z)\left((t+u)_{+}^{\kappa}-u_{+}^{\kappa}\right)+G_{2}(z)\left((t+u)_{-}^{\kappa}-u_{-}^{\kappa}\right)\right) M_{\alpha}(d z, d u), \\
\int_{Z} \int_{\mathbb{R}}\left(\begin{array}{ll}
G_{1}(z)(\ln |t+u|-\ln |u|) \\
\left.+G_{2}(z)\left(1_{(0, \infty)}(t+u)-1_{(0, \infty)}(u)\right)\right) M_{\alpha}(d z, d u), & \text { if } \kappa=0,
\end{array}\right.
\end{array}
$$

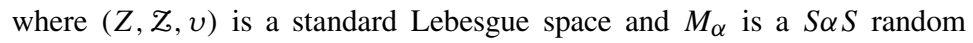
measure on $Z \times \mathbb{R}$ with the control measure $m(d z, d u)=v(d z) d u$. Particular cases include linear fractional stable motions, log-fractional stable motion and $S \alpha S$ Lévy motion. An example of a process $X_{\alpha}^{(3)}$ is

$$
\begin{array}{r}
\int_{0}^{1} \int_{\mathbb{R}}\left((t+u)_{+}^{\kappa} 1_{[0,1 / 2)}(\{x+\ln |t+u|\})\right. \\
\left.-u_{+}^{\kappa} 1_{[0,1 / 2)}(\{x+\ln |u|\})\right) M_{\alpha}(d x, d u),
\end{array}
$$

where $M_{\alpha}$ is a $S \alpha S$ random measure on $[0,1) \times \mathbb{R}$ with the control measure $m(d x, d u)=d x d u$ and $\{\cdot\}$ is the fractional part function.

Received June 2000; revised July 2001.

${ }^{1}$ Supported in part by the NSF Grant ANI-9805623.

AMS 2000 subject classifications. Primary 60G18, 60G52; secondary 28D, 37A.

Key words and phrases. Stable, self-similar processes with stationary increments, dissipative and conservative flows, cocycles, semi-additive functionals. 
1. Introduction. Our goal is to understand the structure of random processes $\left\{X_{\alpha}(t)\right\}_{t \in \mathbb{R}}$ with the following three characteristics: $X_{\alpha}$ is symmetric $\alpha$-stable with $1<\alpha<2$, it is self-similar with index $H \in(0,1)$ and it is a (stationary increments) mixed moving average. We shall first define these terms.

A random variable $\xi$ is symmetric $\alpha$-stable ( $S \alpha S$, in short) with $\alpha \in(0,2]$ if its characteristic function satisfies $E \exp (i \theta \xi)=\exp \left(-\sigma^{\alpha}|\theta|^{\alpha}\right)$ for some scale factor $\sigma>0$ and all $\theta \in \mathbb{R}$. A real-valued stochastic process $\left\{X_{\alpha}(t)\right\}_{t \in \mathbb{R}}$ is $S \alpha S$ with $\alpha \in(0,2]$ if all its linear combinations are $S \alpha S$ random variables. The most common way to represent a $S \alpha S$ process $X_{\alpha}$ is through the so-called spectral representation

$$
\left\{X_{\alpha}(t)\right\}_{t \in \mathbb{R}} \stackrel{d}{=}\left\{\int_{S} f_{t}(s) M_{\alpha}(d s)\right\}_{t \in \mathbb{R}},
$$

where $\stackrel{d}{=}$ stands for the equality in the sense of the finite-dimensional distributions [see Samorodnitsky and Taqqu (1994)]. Here, $M_{\alpha}$ is a $S \alpha S$ random measure with the control measure $m,(S, m)$ is a measure space and $\left\{f_{t}\right\}_{t \in \mathbb{R}}$ is a collection of deterministic functions. The random integrator $M_{\alpha}(d s), s \in S$, can be viewed heuristically as a sequence of independent stable random variables with scale factor $m(d s)$. The representation (1.1) means that the characteristic function of $X_{\alpha}$ is given by

$$
E \exp \left\{i \sum_{k=1}^{n} \theta_{k} X_{\alpha}\left(t_{k}\right)\right\}=\exp \left\{-\int_{S}\left|\sum_{k=1}^{n} \theta_{k} f_{t_{k}}(s)\right|^{\alpha} m(d s)\right\},
$$

where $\theta_{1}, t_{1}, \ldots, \theta_{n}, t_{n} \in \mathbb{R}$. One must therefore require that $\left\{f_{t}\right\}_{t \in \mathbb{R}} \subset L^{\alpha}(S, m)$. The kernel $f_{t}(s)$ in (1.1) characterizes the process $X_{\alpha}$ and our goal is to understand the possible structures of such kernels when $X_{\alpha}$ is, in addition, self-similar and a (stationary increments) mixed moving average.

The process $X_{\alpha}$ is said to be self-similar with index $H>0$, if, for any $c>0$,

$$
\left\{X_{\alpha}(c t)\right\}_{t \in \mathbb{R}} \stackrel{d}{=}\left\{c^{H} X_{\alpha}(t)\right\}_{t \in \mathbb{R}} .
$$

The condition $1<\alpha<2$ implies $0<H<1$ [Corollary 7.1.11 in Samorodnitsky and Taqqu (1994)].

A $S \alpha S$ process $\left\{X_{\alpha}(t)\right\}_{t \in \mathbb{R}}$ is called a (stationary increments) mixed moving average, if it can be represented as

$$
\left\{X_{\alpha}(t)\right\}_{t \in \mathbb{R}} \stackrel{d}{=}\left\{\int_{X} \int_{\mathbb{R}}(G(x, t+u)-G(x, u)) M_{\alpha}(d x, d u)\right\}_{t \in \mathbb{R}},
$$

where $(X, \mathcal{X}, \mu)$ is some measure space and $S \alpha S$ random measure $M_{\alpha}$ has the control measure $\mu(d x) d u$ on $X \times \mathbb{R}$. We call processes of the form (1.4) (stationary increments) mixed moving averages because, heuristically, if one took 
the derivative of $X_{\alpha}$, one would get a stationary process $\dot{X}_{\alpha}$ with the representation $\int_{X} \int_{\mathbb{R}} \dot{G}(x, t+u) M_{\alpha}(d x, d u)$ which is of the so-called mixed moving average type. The mixed part comes from the presence of the additional variable $x$ in the representation of $\dot{X}_{\alpha}$; without $x, \dot{X}_{\alpha}$ would be the usual moving average. The class of (stationary) mixed moving averages was introduced by Surgailis, Rosiński, Mandrekar and Cambanis (1993) as an important extension of the usual moving averages. It is closed under linear combinations and the elements of the class are ergodic. This class also appears in the decomposition of stationary symmetric stable processes established by Rosiński (1995).

One example of processes with the above characteristics is linear fractional stable motion [see Samorodnitsky and Taqqu (1994)] given by

$$
\int_{\mathbb{R}}\left\{a\left((t+u)_{+}^{H-1 / \alpha}-u_{+}^{H-1 / \alpha}\right)+b\left((t+u)_{-}^{H-1 / \alpha}-u_{-}^{H-1 / \alpha}\right)\right\} M_{\alpha}(d u),
$$

where $a, b \in \mathbb{R}, \alpha \in(0,2), H \in(0,1), u_{+}=\max \{u, 0\}, u_{-}=\max \{-u, 0\}$ and $S \alpha S$ random measure $M_{\alpha}$ has the Lebesgue control measure on $\mathbb{R}$. To obtain (1.5) from (1.4), take $Y=\{1\}, \mu(d x)=\delta_{\{1\}}(d x)$ and $G(1, d u)=a u_{+}^{H-1 / \alpha}+b u_{-}^{H-1 / \alpha}$. Another example is the limit of renewal reward processes [see Pipiras and Taqqu (2000)] represented as

$$
\int_{\mathbb{R}} \int_{\mathbb{R}}\left\{(t \wedge z-v)_{+}-(0 \wedge z-v)_{+}\right\}(z-v)_{+}^{H-2 / \alpha-1} M_{\alpha}(d z, d v),
$$

where $M_{\alpha}$ is a $S \alpha S$ random measure on $\mathbb{R}^{2}$ with the Lebesgue control measure $m(d z, d v)=d z d v$. To obtain (1.6), take $X=\mathbb{R}, \mu(d x)=d x$ and $G(x, u)=$ $(u \wedge 0+x)_{+} x_{+}^{H-2 / \alpha-1}$ in (1.4), and then make a change of variables $u=-z, x=$ $z-v$. We showed in Pipiras and Taqqu (2000) that the process (1.6) is not a linear fractional stable motion in (1.5). Recall that, in contrast to the Gaussian case $(\alpha=2)$ where fractional Brownian motion is the only $H$-self-similar process with stationary increments, there are many different $H$-self-similar processes with stationary increments in the stable case $\alpha \in(0,2)$. [See Samorodnitsky and Taqqu (1994) for additional examples.]

We are interested in the following problem:

Is there a way to somehow classify and characterize all stable self-similar processes with stationary increments which have the representation (1.4)?

We assume that the mixing space $X$ in (1.4) is the so-called standard Lebesgue space with a $\sigma$-algebra $\mathcal{X}$ and a $\sigma$-finite measure $\mu$, and that $S \alpha S$ measure $M_{\alpha}$ has the control measure $m(d x, d u)=\mu(d x) d u$ on $X \times \mathbb{R}$. Recall that a standard Lebesgue space is a standard Borel space with a $\sigma$-finite measure and that a standard Borel space is a measurable space measurably isomorphic (i.e., there is a one-to-one, onto and bimeasurable map) to a Borel subset of a complete separable metric space. For example, $\mathbb{R}^{n}$ with a measure consisting 
of Lebesgue measure and discrete point masses is a standard Lebesgue space. We also implicitly assume that $G: X \times \mathbb{R} \mapsto \mathbb{R}$ is a measurable function and that $\left\{G_{t}\right\}_{t \in \mathbb{R}} \subset L^{\alpha}(X \times \mathbb{R}, \mu(d x) d u)$, where $G_{t}(x, u)=G(x, t+u)-G(x, u)$, $t, u \in \mathbb{R}, x \in X$, so that the process $X_{\alpha}$ in (1.4) is well-defined. Observe also that stationarity of the increments is already embedded in the representation (1.4) whereas self-similarity requires additional assumptions on $G$.

To answer the above question, we will use results obtained in Pipiras and Taqqu (2001a). In that paper, we showed that, if $\alpha \in(1,2)$, every self-similar stationary increments mixed moving average $X_{\alpha}$ is generated by a nonsingular measurable multiplicative flow and by a related cocycle and a semi-additive functional (see Section 2 below). Then, by using the so-called Hopf's decomposition of a flow into its dissipative and conservative parts, we established a unique decomposition in distribution of $X_{\alpha}$ into two independent processes

$$
X_{\alpha} \stackrel{d}{=} X_{\alpha}^{D}+X_{\alpha}^{C}
$$

where the process $X_{\alpha}^{D}$ is generated by a nonsingular dissipative flow and $X_{\alpha}^{C}$ is generated by a nonsingular conservative flow. In this paper, we study in greater detail the two components of the decomposition (1.7). This will allow us to obtain a more refined decomposition than (1.7).

The paper is organized as follows. In Section 2, we recall some basic definitions from Pipiras and Taqqu (2001a) which will be used here. Section 3, where we describe cocycles and semi-additive functionals for various types of flows, is of a technical nature. In Section 4 , we show that processes $X_{\alpha}$, generated by dissipative flows, can be completely characterized. Any such process can be represented in distribution as

$$
\int_{Y} \int_{\mathbb{R}} \int_{\mathbb{R}} e^{-\kappa s}\left(F\left(y, e^{s}(t+u)\right)-F\left(y, e^{s} u\right)\right) M_{\alpha}(d y, d s, d u),
$$

where $(Y, \mathcal{Y}, v)$ is some standard Lebesgue space, $F: Y \times \mathbb{R} \mapsto \mathbb{R}$ is a measurable function, $M_{\alpha}$ is a $S \alpha S$ random measure on $Y \times \mathbb{R} \times \mathbb{R}$ with the control measure $v(d y) d s d u$ and $\kappa=H-1 / \alpha$ (see Theorem 4.1). We also mention some examples which, together with a further study of processes (1.8), can be found in Pipiras and Taqqu (2001b).

In Sections 5-8, we consider processes generated by conservative flows. According to Theorem 5.1 in Section 5, any process $X_{\alpha}$, generated by the simplest conservative flow, namely an identity flow, can be represented in distribution as

$$
\begin{aligned}
\int_{Z} \int_{\mathbb{R}}( & G_{1}(z)\left((t+u)_{+}^{\kappa}-u_{+}^{\kappa}\right) \\
& \left.+G_{2}(z)\left((t+u)_{-}^{\kappa}-u_{-}^{\kappa}\right)\right) M_{\alpha}(d z, d u) \quad \text { if } \kappa \neq 0,
\end{aligned}
$$




$$
\begin{aligned}
& \int_{Z} \int_{\mathbb{R}}\left(G_{1}(z)(\ln |t+u|-\ln |u|)\right. \\
&\left.\quad+G_{2}(z)\left(1_{(0, \infty)}(t+u)-1_{(0, \infty)}(u)\right)\right) M_{\alpha}(d z, d u) \quad \text { if } \kappa=0,
\end{aligned}
$$

where $(Z, \mathcal{Z}, v)$ is a standard Lebesgue space, $G_{1}, G_{2}: Z \mapsto \mathbb{R}$ are some functions and a $S \alpha S$ random measure $M_{\alpha}$ has the control measure $v(d z) d u$. We call processes (1.9) mixed linear fractional stable motions (mixed LFSM, in short) because the usual linear fractional stable motions (i.e., when $Z=\{1\}$ and $v=\delta_{\{1\}}$ ) are special cases of processes (1.9). Since processes (1.10) can be viewed as limiting cases of processes (1.9) when $\kappa \rightarrow 0$ (for details, see Definition 5.1 below), we shall call them mixed LFSM as well. Note that the usual log-fractional stable motion (when $Z=\{1\}, v=\delta_{\{1\}}$ and $G_{2} \equiv 0$ ) and the stable Lévy motion (when $Z=\{1\}, v=\delta_{\{1\}}$ and $G_{1} \equiv 0$ ) are special cases of (1.10).

In Sections 6 and 7, we decompose a process generated by a conservative flow into a mixed LFSM and, in analogy to Rosiński (1995), a process of the "third kind." An example of a process of the "third kind" is given in Section 8.

In Section 9, we obtain a decomposition of self-similar processes with stationary increments, having the representation (1.4). This is in the spirit of the decomposition obtained by Rosiński in the context of stationary processes. By using (1.7) and results from Sections 4-8, we show that, for any $\alpha \in$ $(1,2), H>0$, the $H$-self-similar process $X_{\alpha}$ with stationary increments, having the representation (1.4), can be uniquely decomposed in distribution into three independent processes

$$
X_{\alpha} \stackrel{d}{=} X_{\alpha}^{(1)}+X_{\alpha}^{(2)}+X_{\alpha}^{(3)} .
$$

The process $X_{\alpha}^{(1)}$ in (1.11) is the first process $X_{\alpha}^{D}$ in the decomposition (1.7). It is generated by a dissipative flow and, by results of Section 4 , has the representation (1.8). The sum of the two processes $X_{\alpha}^{(2)}$ and $X_{\alpha}^{(3)}$ in (1.11) is the second process $X_{\alpha}^{C}$ in the decomposition (1.7). The process $X_{\alpha}^{(2)}$ has the representation (1.9) or (1.10), depending on whether $\kappa \neq 0$ or $\kappa=0$, that is, it is a mixed LFSM. The process $X_{\alpha}^{(3)}$ is $H$-self-similar process with stationary increments, generated by a conservative flow, which does not have a mixed LFSM component. An example is given in Section 8.

The decomposition (1.11), which is not the same as that of Rosiński, allows one to make a finer distinction between processes. For example, both linear fractional stable motion (1.5) and the limit (1.6) of the renewal reward processes, were they to be differentiable, would have a derivative which is dissipative according to Rosiński's decomposition. According to our decomposition however, while linear fractional stable motion is conservative, the limit of the renewal reward processes is dissipative. This allows us to distinguish these two processes and conclude, as noted in Pipiras and Taqqu (2001a), that they have different finite-dimensional distributions. 
Finally, in Section 10, we draw connections between processes $X_{\alpha}^{(i)}, i=1,2,3$, and the underlying flow in the decomposition (1.11) when the representation (1.4) of the process $X_{\alpha}$ is minimal.

REMARK. When we investigate the processes $X_{\alpha}^{D}$ and $X_{\alpha}^{C}$ in the decomposition (1.7), we assume $\alpha \in(0,2)$. The restriction $\alpha \in(1,2)$ appears only in Sections 7 and 9, where we refine the decomposition (1.7) to get (1.11). As argued in the remark following Theorem 4.2 of Pipiras and Taqqu (2001a), the decomposition (1.7) is still valid when $\alpha \in(0,1]$, provided that the integral $\int_{-\infty}^{t} e^{-(t-s)} X_{\alpha}(s) d s$ is well-defined. Were the decomposition (1.7) valid for $\alpha \in(0,1]$ in general, the results of the present paper would extend to $\alpha \in(0,2)$ as well.

2. Multiplicative flows. We first recall some basic definitions from Pipiras and Taqqu (2001a). Let $(X, \mathcal{X}, \mu)$ be a standard Lebesgue space, as defined in Section $1, \alpha \in(0,2)$ and $H>0$. Set also

$$
\kappa=H-\frac{1}{\alpha} .
$$

If the mixed moving average (1.4) is self-similar, then

$$
\begin{aligned}
\left\{c^{-H}\right. & \left.X_{\alpha}(c t)\right\}_{t \in \mathbb{R}} \\
= & \left\{\int_{X} \int_{\mathbb{R}} c^{-\kappa}(G(x, c(t+u))-G(x, c u)) c^{-1 / \alpha} M_{\alpha}(d x, d c u)\right\}_{t \in \mathbb{R}} \\
& \stackrel{d}{=}\left\{\int_{X} \int_{\mathbb{R}} c^{-\kappa}(G(x, c(t+u))-G(x, c u)) M_{\alpha}(d x, d u)\right\}_{t \in \mathbb{R}} \\
& \stackrel{d}{=}\left\{\int_{X} \int_{\mathbb{R}}(G(x, t+u)-G(x, u)) M_{\alpha}(d x, d u)\right\}_{t \in \mathbb{R}} \\
= & \left\{X_{\alpha}(t)\right\}_{t \in \mathbb{R}} .
\end{aligned}
$$

But while mixed moving averages, as defined by (1.4), are always $S \alpha S$ and have stationary increments, they are not necessarily self-similar. We need to choose $G$ such that $X_{\alpha}$ defined by (1.4) is self-similar and we also want to associate a flow to the process $X_{\alpha}$. The following definition achieves these two goals.

Definition 2.1. A $S \alpha S, H$-self-similar process $X_{\alpha}$ with stationary increments, having the representation (1.4), is said to be generated by a nonsingular measurable multiplicative flow $\left\{\psi_{c}\right\}_{c>0}$ on $(X, \mathcal{X}, \mu)$ associated with $G$ (or 
simply generated by a flow $\left.\left\{\psi_{c}\right\}_{c>0}\right)$ if, for all $c>0$ and $t \in \mathbb{R}$,

$$
\begin{aligned}
c^{-\kappa}( & G(x, c(t+u))-G(x, c u)) \\
= & b_{c}(x)\left\{\frac{d\left(\mu \circ \psi_{c}\right)}{d \mu}(x)\right\}^{1 / \alpha} \\
& \times\left(G\left(\psi_{c}(x), t+u+g_{c}(x)\right)-G\left(\psi_{c}(x), u+g_{c}(x)\right)\right)
\end{aligned}
$$

a.e. $\mu(d x) d u$, where $\left\{b_{c}\right\}_{c>0}$ and $\left\{g_{c}\right\}_{c>0}$ is a cocycle and a semi-additive functional for the flow $\left\{\psi_{c}\right\}_{c>0}$, respectively, and

$$
\operatorname{supp}\{G(x, t+u)-G(x, u), t \in \mathbb{R}\}=X \times \mathbb{R}, \quad \text { a.e. } \mu(d x) d u .
$$

We first explain some of the terms used in this definition and then provide some insight in subsequent remarks. A family $\left\{\psi_{c}\right\}_{c>0}$ of measurable maps from $X$ onto $X$ is called a multiplicative flow if $\psi_{1}(x)=x$ and

$$
\psi_{c_{1}}\left(\psi_{c_{2}}(x)\right)=\psi_{c_{1} c_{2}}(x),
$$

for all $c_{1}, c_{2}>0$ and $x \in X$. Note that relations (2.5) and $\psi_{1}(x)=x$ imply that $\psi_{c}$ has an inverse $\psi_{1 / c}$. [In the literature, one typically considers additive flows $\left\{\phi_{t}\right\}_{t \in \mathbb{R}}$ which satisfy the property $\phi_{t_{1}}\left(\phi_{t_{2}}(x)\right)=\phi_{t_{1}+t_{2}}(x)$, for all $t_{1}, t_{2} \in \mathbb{R}$ and $x \in X$. Physically, think of a particle in position $x$ at time $t=0$. Then $\phi_{t}(x)$ is the position of the particle at time $t$. To go from multiplicative to additive flows, one sets $\phi_{t}=\psi_{e^{t}}$.] It is said to be nonsingular if $\mu\left(\psi_{c}^{-1}(B)\right)=0$ if and only if $\mu(B)=0$, for every $c>0$ and $B \in \mathcal{X}$, and measurable if the map $\psi_{c}(x):(0, \infty) \times X \mapsto X$ is measurable. [Nonsingularity of the map $\psi_{c}$ also means that this map is invertible and that its inverse is measurable. In the case of a flow, these two conditions follow from (2.5), since the inverse of $\psi_{c}$ is a measurable map $\psi_{1 / c}$.] A measurable map $b_{c}(x):(0, \infty) \times X \mapsto\{-1,1\}$ is said to be a cocycle for a flow $\left\{\psi_{c}\right\}_{c>0}$ if

$$
b_{c_{1} c_{2}}(x)=b_{c_{1}}(x) b_{c_{2}}\left(\psi_{c_{1}}(x)\right),
$$

for all $c_{1}, c_{2}>0$ and $x \in X$. A measurable map $g_{c}(x):(0, \infty) \times X \mapsto \mathbb{R}$ is said to be a semi-additive functional for a flow $\left\{\psi_{c}\right\}_{c>0}$ if

$$
g_{c_{1} c_{2}}(x)=\frac{g_{c_{1}}(x)}{c_{2}}+g_{c_{2}}\left(\psi_{c_{1}}(x)\right),
$$

for all $c_{1}, c_{2}>0$ and $x \in X$. The support $\operatorname{supp}\{G(x, t+u)-G(x, u), t \in \mathbb{R}\}$ in (2.4) is defined as the minimal (in the a.e. sense) set $A \in \mathcal{X} \otimes \mathscr{B}$ such that, for all $t \in \mathbb{R}, G(x, t+u)-G(x, u)=0$ for $(x, u) \in X \times \mathbb{R} \backslash A$ a.e. $\mu(d x) d u$.

REMARK 1. To get a feeling for condition (2.3), note that it implies the $H$-self-similarity of the process $X_{\alpha}$. Indeed, for any $\theta_{1}, \ldots, \theta_{n} \in \mathbb{R}, t_{1}, \ldots, t_{n} \in \mathbb{R}$, 
$n \geq 1$ and $c>0$

$$
\begin{aligned}
\int_{X} \int_{\mathbb{R}}\left|\sum_{k=1}^{n} \theta_{k} c^{-\kappa}\left(G\left(x, c\left(t_{k}+u\right)\right)-G(x, c u)\right)\right|^{\alpha} \mu(d x) d u \\
=\int_{X} \int_{\mathbb{R}}\left|\sum_{k=1}^{n} \theta_{k}\left(G\left(\psi_{c}(x), t_{k}+u+g_{c}(x)\right)-G\left(\psi_{c}(x), u+g_{c}(x)\right)\right)\right|^{\alpha} \\
\quad \times \frac{d\left(\mu \circ \psi_{c}\right)}{d \mu}(x) \mu(d x) d u \\
=\int_{X} \int_{\mathbb{R}}\left|\sum_{k=1}^{n} \theta_{k}\left(G\left(\psi_{c}(x), t_{k}+u+g_{c}(x)\right)-G\left(\psi_{c}(x), u+g_{c}(x)\right)\right)\right|^{\alpha} \\
\quad \times\left(\mu \circ \psi_{c}\right)(d x) d u \\
=\int_{X} \int_{\mathbb{R}}\left|\sum_{k=1}^{n} \theta_{k}\left(G\left(x, t_{k}+u\right)-G(x, u)\right)\right|^{\alpha} \mu(d x) d u,
\end{aligned}
$$

since $\psi_{c}(X)=X$, which proves (2.2).

REMARK 3. The condition (2.3) is equivalent to

$$
\begin{aligned}
c^{-\kappa} G(x, c u)= & b_{c}(x)\left\{\frac{d\left(\mu \circ \psi_{c}\right)}{d \mu}(x)\right\}^{1 / \alpha} G\left(\psi_{c}(x), u+g_{c}(x)\right) \\
& +J(x, c) \quad \text { a.e. } \mu(d x) d u
\end{aligned}
$$

where $J: X \times(0, \infty) \mapsto \mathbb{R}$ is some measurable function [Proposition 5.1 in Pipiras and Taqqu (2001a)].

REMARK 4. To understand condition (2.4), suppose on the contrary that the support of the functions $G_{t}(x, u)=G(x, t+u)-G(x, u), t \in \mathbb{R}$, is a set $A \subset X \times \mathbb{R}$ with $A \neq X \times \mathbb{R}$. Because of the mixed moving average structure of the kernel $G_{t}$, there is a set $X_{0} \subset X$ such that $A=X_{0} \times \mathbb{R}$ [see Lemma 4.2 in Pipiras and Taqqu (2001a)]. Condition $A \neq X \times \mathbb{R}$ then translates into $X_{0} \neq X$. Since the functions $G_{t}, t \in \mathbb{R}$, vanish on the complement $\left(X \backslash X_{0}\right) \times \mathbb{R}$ of the support $X_{0} \times \mathbb{R}$, we may in principle define the flow $\left\{\psi_{c}\right\}_{c>0}$ on $X \backslash X_{0}$ arbitrarily because both sides of (2.3) are zero anyway (assuming that $\psi_{c}$ maps $X \backslash X_{0}$ into itself). But this part of the flow would then have nothing to do with the process $X_{\alpha}$. Since we want our flow to capture the underlying structure of the process $X_{\alpha}$, we assume condition (2.4). 
REMARK 5. To get a feeling for (2.5), (2.6) and (2.7), assume that $J \equiv 0$ in (2.8) and let $c_{1}, c_{2}>0$. On one hand, by (2.8),

$$
\begin{aligned}
& \left(c_{1} c_{2}\right)^{-\kappa} G\left(x, c_{1} c_{2} u\right) \\
& \quad=b_{c_{1} c_{2}}(x)\left\{\frac{d\left(\mu \circ \psi_{c_{1} c_{2}}\right)}{d \mu}(x)\right\}^{1 / \alpha} G\left(\psi_{c_{1} c_{2}}(x), u+g_{c_{1} c_{2}}(x)\right) .
\end{aligned}
$$

On the other hand, by iterating (2.8) twice, one gets

$$
\begin{aligned}
\left(c_{1} c_{2}\right)^{-\kappa} G\left(x, c_{1} c_{2} u\right) & \\
= & c_{2}^{-\kappa}\left(c_{1}^{-\kappa} G\left(x, c_{1}\left(c_{2} u\right)\right)\right) \\
= & c_{2}^{-\kappa} b_{c_{1}}(x)\left\{\frac{d\left(\mu \circ \psi_{c_{1}}\right)}{d \mu}(x)\right\}^{1 / \alpha} G\left(\psi_{c_{1}}(x), c_{2}\left(u+c_{2}^{-1} g_{c_{1}}(x)\right)\right) \\
= & b_{c_{1}}(x) b_{c_{2}}\left(\psi_{c_{1}}(x)\right)\left\{\frac{d\left(\mu \circ \psi_{c_{1}}\right)}{d \mu}(x) \frac{d\left(\mu \circ \psi_{c_{2}}\right)}{d \mu}\left(\psi_{c_{1}}(x)\right)\right\}^{1 / \alpha} \\
& \times G\left(\psi_{c_{2}}\left(\psi_{c_{1}}(x)\right), u+c_{2}^{-1} g_{c_{1}}(x)+g_{c_{2}}\left(\psi_{c_{1}}(x)\right)\right) .
\end{aligned}
$$

Relations (2.5), (2.6) and (2.7) imply that the right-hand sides of (2.9) and (2.10) are identical.

Any measurable nonsingular multiplicative flow $\left\{\psi_{c}\right\}_{c>0}$, as in (2.3), can be decomposed into its dissipative and conservative parts. This decomposition is defined as follows [see, e.g., Krengel (1985), page 17, and Rosiński (1995), page 1171]. Consider a single nonsingular map $V: X \rightarrow X$. Then, there exists a unique (in the a.e. sense) decomposition of $X$ into two disjoint sets $C$ and $D$ such that (i) $C$ and $D$ are $V$-invariant, that is, $V C=C$ and $V D=D$, (ii) the restriction of $V$ to $C$ is conservative, that is, there is no set $A \subset C$ such that $\mu(A)>0$ and $V^{-k}(A), k \geq 1$, are disjoint, and (iii) $D=\bigcup_{k=-\infty}^{\infty} V^{k} B$ for some set $B \in \mathcal{X}$, where $V^{-k}(B), k \geq 1$, are disjoint. This is called the Hopf's decomposition of the map $V$. The sets $D$ and $C$ are called the dissipative part and the conservative part, respectively. Since each map $\psi_{c}$ is nonsingular, it has the Hopf decomposition $D_{c}$ and $C_{c}$. One can show [see Krengel (1969), Lemma 2.7] that this decomposition is the same (in the a.e. sense) for all maps $\psi_{c}, c \neq 1$, and that there is a set $D$, invariant under the flow, such that $D=D_{c}, c \neq 1$. Then the decomposition of $X$ into $D$ and $C:=X \backslash D$ is called the Hopf's decomposition of the flow $\left\{\psi_{c}\right\}_{c>0}$. A flow is called dissipative if $X=D$ a.e. and conservative if $X=C$ a.e. In Pipiras and Taqqu (2001a), we used the Hopf's decomposition to establish the representation (1.7). 
3. Cocycles and semi-additive functionals associated with various flows. In this section we describe cocycles and semi-additive functionals for various types of multiplicative flows. (Unless stated otherwise, all the flows are multiplicative.) We first consider a general dissipative flow and then deal with the simplest conservative flow, namely an identity flow. The results we obtain will be used in the following sections.

To deal with dissipative flows, we will often use the following result due to Krengel (1969), page 19 [see also Rosiński (1995), page 1176].

THEOREM 3.1 (Krengel). Every measurable nonsingular dissipative flow $\left\{\psi_{c}\right\}_{c>0}$ on a standard Lebesgue space is null-isomorphic (mod 0) to a flow $\left\{\widetilde{\psi}_{c}\right\}_{c>0}$ on some standard Lebesgue space $(Y \times \mathbb{R}, \mathcal{Y} \otimes \mathcal{B}, v(d y) d u)$ defined by $\widetilde{\psi}_{c}(y, u)=(y, u+\ln c)$ for all $(y, u) \in Y \times \mathbb{R}$ and $c>0$.

A null-isomorphism is a measurable, nonsingular, one-to-one and onto map with a measurable inverse. While it may not be measure preserving, by being nonsingular, it preserves sets of measure zero ("null" refers to nonsingular). Nullisomorphism (mod 0) in Theorem 3.1 means that there exist two null sets $N \subset X$ and $N^{\prime} \subset Y \times \mathbb{R}$, and a null-isomorphism $\Phi: Y \times \mathbb{R} \backslash N^{\prime} \mapsto X \backslash N$ such that

$$
\psi_{c}(\Phi(y, u))=\Phi\left(\widetilde{\psi}_{c}(y, u)\right)
$$

for all $c>0$ and $(y, u) \in Y \times \mathbb{R} \backslash N^{\prime}$. The sets $X \backslash N$ and $Y \times \mathbb{R} \backslash N^{\prime}$ above are invariant under the flows $\left\{\psi_{c}\right\}_{c>0}$ and $\left\{\tilde{\psi}_{c}\right\}_{c>0}$, respectively, that is, $\psi_{c}(X \backslash N)=$ $X \backslash N$ and similarly for $\widetilde{\psi}_{c}$. We will now characterize cocycles and semi-additive functionals related to dissipative flows.

LEMMA 3.1. Let $\left\{\psi_{c}\right\}_{c>0}$ be a dissipative flow, $\left\{g_{c}\right\}_{c>0}$ and $\left\{b_{c}\right\}_{c>0}$ be a semi-additive functional and a cocycle for $\left\{\psi_{c}\right\}_{c>0}$, respectively. Suppose that $\Phi: Y \times \mathbb{R} \backslash N^{\prime} \mapsto X \backslash N$ is a null-isomorphism of Theorem 3.1 between the flows $\left\{\psi_{c}\right\}_{c>0}$ and $\left\{\widetilde{\psi}_{c}\right\}_{c>0}$ described above. Let $\tilde{g}_{c}(y, u)=g_{c}(\Phi(y, u))$, if $(y, u) \in$ $Y \times \mathbb{R} \backslash N^{\prime}$, and $\widetilde{g}_{c}(y, u)=0$, if $(y, u) \in N^{\prime}$, and $\widetilde{b}_{c}(y, u)=b_{c}(\Phi(y, u))$, if $(y, u) \in Y \times \mathbb{R} \backslash N^{\prime}$, and $\widetilde{b}_{c}(y, u)=1$, if $(y, u) \in N^{\prime}$. Then, $\left\{\widetilde{g}_{c}\right\}_{c>0}$ is a semiadditive functional, $\left\{\widetilde{b}_{c}\right\}_{c>0}$ is a cocycle for $\left\{\widetilde{\psi}_{c}\right\}_{c>0}$, and

$$
\widetilde{g}_{c}(y, u)=\widetilde{g}(y, u+\ln c)-\frac{\tilde{g}(y, u)}{c}, \quad \widetilde{b}_{c}(y, u)=\frac{\widetilde{b}(y, u+\ln c)}{\widetilde{b}(y, u)},
$$

for all $c>0$ and $(y, u) \in Y \times \mathbb{R}$, where $\tilde{g}: Y \times \mathbb{R} \mapsto \mathbb{R}$ and $\widetilde{b}: Y \times \mathbb{R} \mapsto\{-1,1\}$ are some measurable functions.

Proof. In view of the invariance of $X \backslash N$ and $Y \times \mathbb{R} \backslash N^{\prime}$ under the flows $\left\{\psi_{c}\right\}_{c>0}$ and $\left\{\widetilde{\psi}_{c}\right\}_{c>0}$, respectively, we may, without loss of generality, suppose that 
$N$ and $N^{\prime}$ are empty sets. We will prove the lemma for semi-additive functionals first. By definition of a semi-additive functional, for all $c_{1}, c_{2}>0$ and $x \in X$,

$$
g_{c_{1} c_{2}}(x)=c_{2}^{-1} g_{c_{1}}(x)+g_{c_{2}}\left(\psi_{c_{1}}(x)\right) .
$$

Then

$$
g_{c_{1} c_{2}}(\Phi(y, u))=c_{2}^{-1} g_{c_{1}}(\Phi(y, u))+g_{c_{2}}\left(\psi_{c_{1}}(\Phi(y, u))\right)
$$

and, since $\psi_{c} \circ \Phi=\Phi \circ \widetilde{\psi}_{c}$, we obtain that

$$
\begin{aligned}
\tilde{g}_{c_{1} c_{2}}(y, u) & =c_{2}^{-1} \widetilde{g}_{c_{1}}(y, u)+\widetilde{g}_{c_{2}}\left(\widetilde{\psi}_{c_{1}}(y, u)\right) \\
& =c_{2}^{-1} \widetilde{g}_{c_{1}}(y, u)+\widetilde{g}_{c_{2}}\left(y, u+\ln c_{1}\right) .
\end{aligned}
$$

The relation (3.1) shows that $\widetilde{g}_{c}(y, u)=g_{c}(\Phi(y, u))$ is a semi-additive functional for $\left\{\widetilde{\psi}_{c}\right\}_{c>0}$. Moreover, by taking $u=0$ in (3.1), we get that

$$
\widetilde{g}_{c_{1} c_{2}}(y, 0)=c_{2}^{-1} \widetilde{g}_{c_{1}}(y, 0)+\widetilde{g}_{c_{2}}\left(y, \ln c_{1}\right)
$$

or, by letting $\ln c_{1}=v$ and $c_{2}=c$, that

$$
\widetilde{g}_{c}(y, v)=\widetilde{g}_{e^{v+\ln c}}(y, 0)-c^{-1} \widetilde{g}_{e^{v}}(y, 0)=\widetilde{g}(y, v+\ln c)-c^{-1} \widetilde{g}(y, v),
$$

where $\tilde{g}(y, s):=\tilde{g}_{e^{s}}(y, 0)$. Although $\tilde{g}_{c}(y, s)$ is measurable in $c, y$ and $s$, we need to show that it is measurable in $c$ and $y$ when $s=0$. To do so, set $u=-\ln c_{1}$ in (3.1). Then $\widetilde{g}_{c_{2}}(y, 0)=\widetilde{g}_{c_{1} c_{2}}\left(y,-\ln c_{1}\right)-c_{2}^{-1} \widetilde{g}_{c_{1}}\left(y,-\ln c_{1}\right)=: \widetilde{g}\left(c_{1}, c_{2}, y\right)$ and the function $\tilde{g}\left(c_{1}, c_{2}, y\right)$ is measurable in its arguments. Hence, by fixing $c_{1}$ for which it is measurable in $\left(c_{2}, y\right)$, we obtain the measurability of $\widetilde{g}_{c_{2}}(y, 0)$.

Let us now turn to cocycles. Since

$$
\begin{aligned}
\widetilde{b}_{c_{1} c_{2}}(y, u)=b_{c_{1} c_{2}}(\Phi(y, u)) & =b_{c_{1}}(\Phi(y, u)) b_{c_{2}}\left(\psi_{c_{1}}(\Phi(y, u))\right) \\
& =b_{c_{1}}(\Phi(y, u)) b_{c_{2}}\left(\Phi\left(\widetilde{\psi}_{c_{1}}(y, u)\right)\right. \\
& =b_{c_{1}}(\Phi(y, u)) b_{c_{2}}\left(\Phi\left(y, u+\ln c_{1}\right)\right) \\
& =\widetilde{b}_{c_{1}}(y, u) \widetilde{b}_{c_{2}}\left(y, u+\ln c_{1}\right),
\end{aligned}
$$

$\left\{\tilde{b}_{c}\right\}_{c>0}$ is a cocycle for $\left\{\widetilde{\psi}_{c}\right\}_{c>0}$, and, by letting $u=0, c_{2}=c$ and $\ln c_{1}=v$, we get

$$
\widetilde{b}_{c}(y, v)=\frac{\widetilde{b}_{e^{v+\ln c}}(y, 0)}{\widetilde{b}_{e^{v}}(y, 0)}=\frac{\widetilde{b}(y, v+\ln c)}{\widetilde{b}(y, v)},
$$

where $\widetilde{b}(y, u)=\widetilde{b}_{e^{u}}(y, 0)$. [One may argue that the function $\widetilde{b}(y, u)$ is measurable as in the case above of the semi-additive functional.]

We now turn to identity flows which are the simplest of conservative flows. A flow $\left\{\psi_{c}\right\}_{c>0}$ on a space $(X, \mathcal{X}, \mu)$ is called an identity flow if $\psi_{c}(x)=x$, for all $c>0$ and $x \in X$. In the following lemma we characterize semi-additive functionals and cocycles for an identity flow. 
LEMmA 3.2. Let $\left\{\psi_{c}\right\}_{c>0}$ be an identity flow on a space $X$. If $\left\{b_{c}\right\}_{c>0}$ is a cocycle and $\left\{g_{c}\right\}_{c>0}$ is a semi-additive functional for the flow $\left\{\psi_{c}\right\}_{c>0}$, then

$$
b_{c}(x)=1, \quad g_{c}(x)=\left(c^{-1}-1\right) g(x),
$$

for all $c>0$ and $x \in X$, where $g: X \mapsto \mathbb{R}$ is a measurable map.

PROOF. The proof is straightforward. Since $\left\{b_{c}\right\}_{c>0}$ is a cocycle for an identity flow, by (2.6), $b_{c_{1} c_{2}}(x)=b_{c_{1}}(x) b_{c_{2}}(x)$ for all $c>0$ and $x \in X$. In particular, $b_{c}(x)=b_{\sqrt{c}}(x) b_{\sqrt{c}}(x)=\left(b_{\sqrt{c}}(x)\right)^{2}$ and, since $b_{\sqrt{c}}(x) \in\{-1,1\}, b_{c}(x)=1$. For the semi-additive functional $\left\{g_{c}\right\}_{c>0}$, we have

$$
g_{c_{1} c_{2}}(x)=c_{2}^{-1} g_{c_{1}}(x)+g_{c_{2}}(x)=c_{1}^{-1} g_{c_{2}}(x)+g_{c_{1}}(x),
$$

for all $c_{1}, c_{2}>0$ and $x \in X$. This implies

$$
\left(c_{2}^{-1}-1\right) g_{c_{1}}(x)=\left(c_{1}^{-1}-1\right) g_{c_{2}}(x),
$$

or, by letting $c_{1}=c$ and $c_{2}=e$,

$$
g_{c}(x)=\left(c^{-1}-1\right) g(x),
$$

where $g(x)=\left(e^{-1}-1\right)^{-1} g_{e}(x)$.

4. Processes generated by dissipative flows. In the next theorem, we show that $S \alpha S$ processes generated by dissipative multiplicative flows have a canonical representation.

THEOREM 4.1. Let $\alpha \in(0,2), H>0$ and $\kappa=H-1 / \alpha$. Let also $X_{\alpha}$ be a $S \alpha S$ processes generated by a dissipative multiplicative flow as in Definition 2.1. Then there is a standard Lebesgue space $(Y, y, v)$ and a measurable function $F: Y \times \mathbb{R} \mapsto \mathbb{R}$ such that

$$
\begin{aligned}
\left\{X_{\alpha}(t)\right\}_{t \in \mathbb{R}} \stackrel{d}{=}\left\{\int_{Y} \int_{\mathbb{R}} \int_{\mathbb{R}} e^{-\kappa s}\left(F\left(y, e^{s}(t+u)\right)-F\left(y, e^{s} u\right)\right)\right. \\
\left.\times M_{\alpha}(d y, d s, d u)\right\}_{t \in \mathbb{R}},
\end{aligned}
$$

where $M_{\alpha}$ is a $S \alpha S$ random measure on $Y \times \mathbb{R} \times \mathbb{R}$ with the control measure $m(d y, d s, d u)=v(d y) d s d u$. Conversely, if the process $X_{\alpha}$ has the representation (4.1), then it is generated by a dissipative flow.

Proof. According to Theorem 3.1, there is a standard Lebesgue space $(Y \times$ $\mathbb{R}, \mathcal{y} \times \mathcal{R}, v(d y) d s)$ and a null-isomorphism $\Phi: Y \times \mathbb{R} \mapsto X$ such that

$$
\psi_{c}(x)=\psi_{c}(\Phi(y, s))=\Phi(y, s+\ln c),
$$


for all $c>0$ and $(y, s) \in Y \times \mathbb{R}$. In other words, the flow $\left\{\psi_{c}\right\}_{c>0}$ on $(X, \mathcal{X}, \mu)$ is null-isomorphic to a flow $\left\{\widetilde{\psi}_{c}\right\}_{c>0}$ on $(Y \times \mathbb{R}, v(d y) d s)$ defined by $\widetilde{\psi}_{c}(y, s)=$ $(y, s+\ln c)$. [We may suppose that the null sets in Theorem 3.1 are empty because, otherwise, we can replace $X$ by $X \backslash N$ in the definition (1.4) of $X_{\alpha}$ without changing its distribution.] By replacing $x$ by $\Phi(y, s)$ in (2.8) and using (4.2), we get for all $c>0$

$$
c^{-\kappa} G(\Phi(y, s), c u)=b_{c}(\Phi(y, s))\left\{\frac{d\left(\mu \circ \psi_{c}\right)}{d \mu}(\Phi(y, s))\right\}^{1 / \alpha}
$$

$$
\times G\left(\Phi(y, s+\ln c), u+g_{c}(\Phi(y, s))\right)+J(\Phi(y, s), c)
$$

a.e. $v(d y) d s d u$. By Lemma 3.1, we also have that a cocycle $\left\{\widetilde{b}_{c}\right\}_{c>0}=\left\{b_{c} \circ \Phi\right\}_{c>0}$ and a semi-additive functional $\left\{\widetilde{g}_{c}\right\}_{c>0}=\left\{g_{c} \circ \Phi\right\}_{c>0}$ for the multiplicative flow $\left\{\widetilde{\psi}_{c}\right\}_{c>0}$ can be expressed as

$$
\begin{aligned}
& b_{c}(\Phi(y, s))=\widetilde{b}(y, s+\ln c)(\widetilde{b}(y, s))^{-1}, \\
& g_{c}(\Phi(y, s))=\widetilde{g}(y, s+\ln c)-\frac{\widetilde{g}(y, s)}{c}
\end{aligned}
$$

for some measurable functions $\widetilde{b}$, taking values in $\{-1,1\}$, and $\widetilde{g}$. Moreover, we have that ( $\mathbb{L}$ denotes the Lebesgue measure)

$$
\begin{aligned}
& \frac{d\left(\mu \circ \psi_{c}\right)}{d \mu}(\Phi(y, s)) \\
& \quad=\frac{d(\mu \circ \Phi)}{d(v \otimes \mathbb{L})}\left(\widetilde{\psi}_{c}(y, s)\right)\left\{\frac{d \mu}{d(v \otimes \mathbb{L}) \circ \Phi^{-1}}(\Phi(y, s))\right\}^{-1} .
\end{aligned}
$$

To show (4.4), observe first that, since $\psi_{c} \circ \Phi=\Phi \circ \widetilde{\psi}_{c}$, we have

$$
\frac{d\left(\mu \circ \psi_{c} \circ \Phi\right)}{d(v \otimes \mathbb{L})}=\frac{d\left(\mu \circ \Phi \circ \widetilde{\psi}_{c}\right)}{d(v \otimes \mathbb{L})}
$$

Relation (4.4) is merely a different expression for (4.5), since on one hand,

$$
\begin{aligned}
\frac{d\left(\mu \circ \psi_{c} \circ \Phi\right)}{d(v \otimes \mathbb{L})} & =\frac{d\left(\mu \circ \psi_{c} \circ \Phi\right)}{d(\mu \circ \Phi)} \frac{d(\mu \circ \Phi)}{d(v \otimes \mathbb{L})} \\
& =\frac{d\left(\mu \circ \psi_{c}\right)}{d \mu} \circ \Phi \frac{d \mu}{d(v \otimes \mathbb{L}) \circ \Phi^{-1}} \circ \Phi,
\end{aligned}
$$

and on the other hand,

$$
\frac{d\left(\mu \circ \Phi \circ \widetilde{\psi}_{c}\right)}{d(v \otimes \mathbb{L})}=\frac{d(v \otimes \mathbb{L}) \circ \widetilde{\psi}_{c}}{d(v \otimes \mathbb{L})} \frac{d\left(\mu \circ \Phi \circ \widetilde{\psi}_{c}\right)}{d(v \otimes \mathbb{L}) \circ \widetilde{\psi}_{c}}=\frac{d(\mu \circ \Phi)}{d(v \otimes \mathbb{L})} \circ \widetilde{\psi}_{c},
$$


where $d\left((v \otimes \mathbb{L}) \circ \widetilde{\psi}_{c}\right) / d(v \otimes \mathbb{L})=1$ because the first component in $\widetilde{\psi}_{c}(y, s)=$ $(y, s+\ln c)$ remains the same and the second is a translation. Now, by setting

$$
\widetilde{G}(y, s, u)=\widetilde{b}(y, s)\left\{\frac{d \mu}{d(v \otimes \mathbb{L}) \circ \Phi^{-1}}(\Phi(y, s))\right\}^{1 / \alpha} G(\Phi(y, s), u)
$$

and

$$
\widetilde{F}(y, s, u)=\tilde{b}(y, s)\left\{\frac{d(\mu \circ \Phi)}{d(v \otimes \mathbb{L})}(y, s)\right\}^{1 / \alpha} G(\Phi(y, s), u)
$$

in (4.3), we obtain that, for all $c>0$,

$$
c^{-\kappa} \widetilde{G}(y, s, c u)=\widetilde{F}\left(y, s+\ln c, u+\widetilde{g}(y, s+\ln c)-\frac{\tilde{g}(y, s)}{c}\right)+\widetilde{J}(y, s, c)
$$

a.e. $v(d y) d s d u$, where $\widetilde{J}$ is some measurable function. By making the change of variables $c u=z$, we get

$$
\widetilde{G}(y, s, z)=c^{\kappa} \widetilde{F}\left(y, s+\ln c, \frac{z}{c}+\widetilde{g}(y, s+\ln c)-\frac{\widetilde{g}(y, s)}{c}\right)+c^{\kappa} \widetilde{J}(y, s, c)
$$

a.e. $v(d y) d s d z$. By the Fubini's theorem, this relation holds a.e $v(d y) d s d z d c$ as well. Then, by setting $u=s+\ln c$, we get $c=e^{u-s}$ and

$\widetilde{G}(y, s, z)=e^{\kappa(u-s)} \widetilde{F}\left(y, u, e^{s-u} z+\widetilde{g}(y, u)-e^{s-u} \widetilde{g}(y, s)\right)+e^{(s-u) \kappa} \widetilde{J}\left(y, s, e^{s-u}\right)$ a.e. $v(d y) d s d z d u$. Fix $u=u_{0}$ for which this relation holds a.e. $v(d y) d s d z$. Then

$$
\widetilde{G}(y, s, z)=e^{-\kappa s} F\left(y, e^{s}(z-\widetilde{g}(y, s))\right)+J(y, s),
$$

a.e. $v(d y) d s d z$ for some measurable functions $F$ and $J$. Now, by writing down the characteristic functions, it is easy to see that (4.6) implies

$$
\begin{aligned}
\left\{X_{\alpha}(t)\right\}_{t \in \mathbb{R}} & \stackrel{d}{=}\left\{\int_{X} \int_{\mathbb{R}}(G(x, t+u)-G(x, u)) M_{\alpha}(d x, d u)\right\}_{t \in \mathbb{R}} \\
& \stackrel{d}{=}\left\{\int_{Y} \int_{\mathbb{R}} \int_{\mathbb{R}}(\widetilde{G}(y, s, t+u)-\widetilde{G}(y, s, u)) \widetilde{M}_{\alpha}(d y, d s, d u)\right\}_{t \in \mathbb{R}},
\end{aligned}
$$

where $\widetilde{M}_{\alpha}$ is a $S \alpha S$ random measure on $Y \times \mathbb{R} \times \mathbb{R}$ with the control measure $\tilde{m}(d y, d s, d u)=v(d y) d s d u$. Then, by using (4.7), we get that

$$
\begin{aligned}
& \left\{X_{\alpha}(t)\right\}_{t \in \mathbb{R}} \\
& \quad \stackrel{d}{=}\left\{\int_{Y} \int_{\mathbb{R}} \int_{\mathbb{R}} e^{-\kappa s}\left(F\left(y, e^{s}(t+u)\right)-F\left(y, e^{s} u\right)\right) \widetilde{M}_{\alpha}(d y, d s, d u)\right\}_{t \in \mathbb{R}} .
\end{aligned}
$$

To prove the converse, suppose that the process $X_{\alpha}$ has the representation (4.1). By using an argument similar to the one in the proof of Lemma 4.2 in Pipi- 
ras and Taqqu (2001a), one can conclude that there is a set $Y_{0} \in \mathcal{y}$ such that $\operatorname{supp}\left\{e^{-\kappa s}\left(F\left(y, e^{s}(t+u)\right)-F\left(y, e^{s} u\right)\right), t \in \mathbb{R}\right\}=Y_{0} \times \mathbb{R} \times \mathbb{R}$ a.e. $v(d y) d s d u$. Hence, by replacing $Y$ with $Y_{0}$ in (4.1), we may suppose that the condition (2.4) holds. We may do this without loss of generality because this replacement does not change the distribution of $X_{\alpha}$. We shall now show that relation (2.8) is satisfied with $G(y, s, u)=e^{-\kappa s} F\left(y, e^{s} u\right)$, where the $x$ in this relation stands for $(y, s)$. Observe that, since $c^{-\kappa} G(y, s, c u)=G(y, s+\ln c, u)$ for any $c>0$, the condition (2.8) is satisfied with $\psi_{c}(y, s)=(y, s+\ln c), b_{c}(y, s)=1, g_{c}(y, s)=0$ and $J(y, s, c)=0$. One still needs to verify that $\left\{\psi_{c}\right\}_{c>0}$ is a dissipative multiplicative flow. It is obviously a (measurable, nonsingular) multiplicative flow. To see why it is dissipative, recall the definition given at the end of Section 2 and observe that, say for $c>1, \psi_{c}(y, s)=(y, s+\ln c)$, the sets $\psi_{c}^{k}(Y \times[0, \ln c))=Y \times[k \ln c$, $(k+1) \ln c), k \in \mathbb{Z}$, are disjoint and $Y \times \mathbb{R}=\cup_{k \in \mathbb{Z}} \psi_{c}^{k}(Y \times[k \ln c,(k+1) \ln c))$.

REMARK. Further study of mixed moving averages generated by dissipative flows can be found in Pipiras and Taqqu (2001b). In particular, we provide there many examples of such processes, for example, the limit process (1.6) of the renewal reward problem discussed above, the so-called "random wavelet expansion" of Chi (2001), the Takenaka process of Takenaka (1991) and the "new" self-similar processes (3.1) of Samorodnitsky and Taqqu (1990).

5. Processes generated by identity flows. We now turn to processes generated by conservative flows, that is, processes $X_{\alpha}^{C}$ in the decomposition (1.7). In contrast to Section 4 , we will not provide a canonical representation of such processes because conservative flows cannot be characterized as simply as dissipative flows are by the Krengel's Theorem 3.1. Instead, in this and the following three sections, we will pursue a different idea. We begin by showing that one can characterize processes generated by the simplest conservative flows, namely, identity flows. Recall that a multiplicative flow $\left\{\psi_{c}\right\}_{c>0}$ is an identity flow on $(X, X, \mu)$ if $\psi_{c}(x)=x$ for all $x \in X$ and $c>0$. The processes that we get will enter in our finer decomposition of the process $X_{\alpha}^{C}$ considered in Section 7 below.

TheOREM 5.1. Let $\alpha \in(0,2)$ and $H>0$. Suppose that the process $X_{\alpha}$ is generated by an identity flow in the sense of Definition 2.1. Then there exist measurable functions $G_{1}, G_{2}: X \mapsto \mathbb{R}$ such that the process $\left\{X_{\alpha}(t)\right\}_{t \in \mathbb{R}}$ can be represented as

$$
\begin{aligned}
\int_{X} \int_{\mathbb{R}}\left(G_{1}(x)\left((t+u)_{+}^{\kappa}-u_{+}^{\kappa}\right)+G_{2}(x)\left((t+u)_{-}^{\kappa}-u_{-}^{\kappa}\right)\right) \\
\times M_{\alpha}(d x, d u) \quad \text { if } \kappa \neq 0
\end{aligned}
$$




$$
\begin{aligned}
\int_{X} \int_{\mathbb{R}}\left(G_{1}(x)(\ln |t+u|-\ln |u|)+G_{2}(x)\left(1_{(0, \infty)}(t+u)-1_{(0, \infty)}(u)\right)\right) \\
\times M_{\alpha}(d x, d u) \quad \text { if } \kappa=0,
\end{aligned}
$$

where $M_{\alpha}$ is a $S \alpha S$ random measure on $X \times \mathbb{R}$ with the control measure $m(d x, d u)=\mu(d x) d u$.

Conversely, if the process $X_{\alpha}$ has representation (5.1) or (5.2) with $\operatorname{supp}\left\{G_{1}, G_{2}\right\}=X$ a.e. $\mu(d x)$, then it is generated by an identity flow.

PROOF. If $\left\{\psi_{c}\right\}_{c>0}$ is an identity flow, then relation (2.8), which is equivalent to (2.3), becomes

$$
c^{-\kappa} G(x, c u)=b_{c}(x) G\left(x, u+g_{c}(x)\right)+J(x, c)
$$

for all $c>0$ a.e. $\mu(d x) d u$. Since $\left\{b_{c}\right\}_{c>0}$ is a cocycle and $\left\{g_{c}\right\}_{c>0}$ is a semiadditive functional for an identity flow, we obtain from Lemma 3.2 that $b_{c}(x)=1$ and $g_{c}(x)=\left(c^{-1}-1\right) g(x)$ for some measurable function $g$. Then, relation (5.3) becomes

$$
c^{-\kappa} G(x, c u)=G\left(x, u+\left(c^{-1}-1\right) g(x)\right)+J(x, c)
$$

for all $c>0$ a.e. $\mu(d x) d u$. As in Cambanis, Maejima and Samorodnitsky [(1992), page 104], it follows from (5.4) that, for all $c_{1}, c_{2}>0$,

$$
J\left(x, c_{1} c_{2}\right)=c_{1}^{-\kappa} J\left(x, c_{2}\right)+J\left(x, c_{1}\right)=c_{2}^{-\kappa} J\left(x, c_{1}\right)+J\left(x, c_{2}\right)
$$

a.e. $\mu(d x)$. We shall now consider the cases $\kappa \neq 0$ and $\kappa=0$ separately.

Case $\kappa \neq 0$. It follows from the second equality in (5.5) that, for all $c_{1}, c_{2}>0$ and $c_{1}, c_{2} \neq 1$,

$$
\frac{J\left(x, c_{1}\right)}{1-c_{1}^{-\kappa}}=\frac{J\left(x, c_{2}\right)}{1-c_{2}^{-\kappa}}
$$

a.e. $\mu(d x)$. Then, for all $c>0, J(x, c)=J(x)\left(1-c^{-\kappa}\right)$ a.e. $\mu(d x)$ for some measurable function $J$. Relation (5.4) now becomes

$$
c^{-\kappa} G(x, c u)=G\left(x, u+\left(c^{-1}-1\right) g(x)\right)+J(x)\left(1-c^{-\kappa}\right)
$$

for all $c>0$ a.e. $\mu(d x) d u$, which by the Fubini's theorem holds also a.e. $\mu(d x) d u d c$. By making a change of variables, we obtain that $G(x, z)=$ $c^{\kappa} G\left(x, c^{-1}(z+g(x))-g(x)\right)+J(x)\left(c^{\kappa}-1\right)$ and hence

$$
\begin{aligned}
G(x, z)+J(x) & =c^{\kappa}\left(G\left(x, c^{-1}(z+g(x))-g(x)\right)+J(x)\right) \\
& =c^{\kappa} F\left(x, c^{-1}(z+g(x))\right)
\end{aligned}
$$

a.e. $\mu(d x) d z \underset{\widetilde{G}}{d}$ for some function $F$. By letting $\widetilde{G}(x, z)=G(x, z-g(x))+J(x)$, we get that $\widetilde{G}(x, z)=c^{\kappa} F\left(x, c^{-1} z\right)$ a.e. $\mu(d x) d z d c$. In particular, $\widetilde{G}(x, z)=$ 
$z^{\kappa}\left(c^{-1} z\right)^{-\kappa} F\left(x, c^{-1} z\right)$ a.e. $\mu(d x) d z d c$ when $z>0$ and hence $\widetilde{G}(x, z)=$ $z^{\kappa} v^{-\kappa} F(x, v)$ a.e. $\mu(d x) d v d z$ when $z>0$. By fixing $v=v_{0}$, for which this equation holds a.e. $\mu(d x) d z$, we get that $\widetilde{G}(x, z)=z^{\kappa} G_{1}(x)$ a.e. $\mu(d x) d z$ for some function $G_{1}$ when $z>0$. Similarly, $\widetilde{G}(x, z)=z_{-}^{\kappa} G_{2}(x)$ a.e. $\mu(d x) d z$ for some function $G_{2}$ when $z<0$. Hence

$$
G(x, z)=G_{1}(x)(z+g(x))_{+}^{\kappa}+G_{2}(x)(z+g(x))_{-}^{\kappa}-J(x)
$$

a.e. $\mu(d x) d z$. The result (5.1) of the theorem now follows.

Case $\kappa=0$. Since the second equality in (5.5) is now trivial, we shall use the first equality instead, namely that, for all $c_{1}, c_{2}>0, J\left(x, c_{1} c_{2}\right)=J\left(x, c_{2}\right)+$ $J\left(x, c_{1}\right)$ a.e. $\mu(d x)$. By setting $k(x, s)=J\left(x, e^{s}\right), s \in \mathbb{R}$, we obtain that, for all $s, t \in \mathbb{R}$,

$$
k(x, s+t)=k(x, s)+k(x, t)
$$

a.e. $\mu(d x)$. It is easy to see that for a.e. $x \in X$, the function $k(x, s), s \in \mathbb{R}$, satisfies the conditions (i)-(iv) of Proposition A.1 in the Appendix. To understand, for example, why the condition (ii) is satisfied, observe that, since $\mathbb{Q}$ is countable, one may first conclude that, for $s \in \mathbb{R}$, the relation (5.8) holds also for a.e. $x \in X$ and for all $t \in \mathbb{Q}$ (the a.e. set here depends on $s \in \mathbb{R}$ only). Then, by using the Fubini's theorem, the relation (5.8) holds also for a.e. $(x, s)$ and for all $t \in \mathbb{Q}$, which now implies that, for a.e. $x \in X$, the function $k(x, s)$ satisfies the condition (ii). Proposition A.1 now implies that for a.e. $x \in X, k(x, s)=k(x, 1) s$ a.e. $d s$. We may suppose without loss of generality that the function $k(x, 1)$ is measurable [otherwise, consider $k_{0}(x, s)=k\left(x, s_{0} s\right)$ where $s_{0}$ is such that $k\left(x, s_{0}\right)$ is measurable]. Therefore, we deduce that for some measurable function $G_{1}: X \rightarrow \mathbb{R}, J(x, c)=k(x, \ln c)=G_{1}(x) \ln c$ a.e. $\mu(d x) d c$. Relation (5.4) can now be written as

$$
G(x, c u)=G\left(x, u+\left(c^{-1}-1\right) g(x)\right)+G_{1}(x) \ln c
$$

a.e. $\mu(d x) d c d u$. By making the change of variables $u+c^{-1} g(x)=v$, we have

$$
G(x, c v-g(x))=G(x, v-g(x))+G_{1}(x) \ln c
$$

or, by setting $\widetilde{G}(x, z)=G(x, z-g(x))$,

$$
\widetilde{G}(x, c v)=\widetilde{G}(x, v)+G_{1}(x) \ln c
$$

a.e. $\mu(d x) d c d v$. Consider now $v>0$ and write the above relation as

$$
\widetilde{G}(x, c v)-G_{1}(x) \ln c v=\widetilde{G}(x, v)-G_{1}(x) \ln v
$$

a.e. $\mu(d x) d c d v$. By setting $\widehat{G}(x, z)=\widetilde{G}(x, z)-G_{1}(x) \ln z$ for $x \in X, z>0$, we then have $\widehat{G}(x, c v)=\widehat{G}(x, v)$ a.e. $\mu(d x) d c d v$. By making the change of variables $c=z / v$ and then fixing $v$, we deduce that $\widehat{G}(x, z)=G_{2,1}(x) 1_{(0, \infty)}(z)$ 
a.e. $\mu(d x) d z$ for some $G_{2,1}$. Going backwards, we get for $z+g(x)>0$,

$$
G(x, z)=\widetilde{G}(x, z+g(x))=G_{2,1}(x) 1_{(0, \infty)}(z+g(x))+G_{1}(x) \ln (z+g(x))
$$

a.e. $\mu(d x) d z$. When $z+g(x)<0$, one may deduce similarly that, for some function $G_{2,2}$,

$$
\begin{aligned}
G(x, z) & =G_{2,2}(x) 1_{(-\infty, 0)}(z+g(x))+G_{1}(x) \ln |z+g(x)| \\
& =G_{2,2}(x)-G_{2,2}(x) 1_{(0, \infty)}(z+g(x))+G_{1}(x) \ln |z+g(x)|
\end{aligned}
$$

a.e. $\mu(d x) d z$. By combining the previous two relations we get

$$
\begin{aligned}
G(x, z)= & \left(G_{2,1}(x)-G_{2,2}(x)\right) 1_{(0, \infty)}(z+g(x)) \\
& +G_{1}(x) \ln |z+g(x)|+G_{2,2}(x)
\end{aligned}
$$

a.e. $\mu(d x) d z$ and hence representation (5.2).

Suppose now that $\kappa \neq 0$ and the process $X_{\alpha}$ has representation (5.1) which is also representation (1.4) with the kernel function $G(x, u)=G_{1}(x) u_{+}^{\kappa}+G_{2}(x) u_{-}^{\kappa}$, $x \in X, u \in \mathbb{R}$. Since $c^{-\kappa} G(x, c u)=G(x, u)$, we see that condition (2.3) holds with the identity flow $\psi_{c}(x)=x$ [and the corresponding cocycle $b_{c}(x)=1$ and the semi-additive functional $g_{c}(x)=0$ ]. To conclude that $X_{\alpha}$ is generated by an identity flow, one still needs to check condition (2.4). Assume that (2.4) does not hold. Then, by Lemma 4.2 in Pipiras and Taqqu (2001a), there is a measurable set $X_{0} \subset X$ with $\mu\left(X_{0}\right)>0$ such that, for all $t \in \mathbb{R}, G(x, t+u)-G(x, u)=0$ a.e. for $x \in X_{0}$ and $u \in \mathbb{R}$. Hence, by the Fubini's theorem and a change of variables, $G(x, v)=G(x, u)$ a.e. for $x \in X_{0}$ and $u, v \in \mathbb{R}$. This condition implies that $G_{1}(x)=0$ and $G_{2}(x)=0$ a.e. for $x \in X_{0}$. Since $\mu\left(X_{0}\right)>0$, this contradicts $\operatorname{supp}\left\{G_{1}, G_{2}\right\}=X$. The case $\kappa=0$ may be proved in a similar way.

REMARK 1. The process (5.1) is well defined for $G_{1}, G_{2} \in L^{\alpha}(X, X, \mu), \alpha \in$ $(0,2)$ and $H \in(0,1)$, since by using the inequality $|a+b|^{\alpha} \leq \operatorname{const}\left(|a|^{\alpha}+|b|^{\alpha}\right)$,

$$
\begin{aligned}
\int_{X} \int_{\mathbb{R}}\left|G_{1}(x)\left((t+u)_{+}^{\kappa}-u_{+}^{\kappa}\right)+G_{2}(x)\left((t+u)_{-}^{\kappa}-u_{-}^{\kappa}\right)\right|^{\alpha} \\
\quad \times \mu(d x) d u<\infty,
\end{aligned}
$$

for all $t \in \mathbb{R}$. It is not defined for $H \geq 1$ since the integral in (5.10) equals $+\infty$. This also means that the process $X_{\alpha}$ cannot be generated by an identity flow when $H \geq 1$.

REMARK 2. The process (5.1) is also a mixed fractional motion in the sense of Burnecki, Rosiński and Weron (1998), since for $t>0$ it can be represented in 
distribution as

$$
\begin{aligned}
& \int_{X} \int_{0}^{\infty} u^{\kappa} G_{1}(x)\left(\left(u^{-1} t+1\right)_{+}^{\kappa}-1\right) M_{\alpha}(d x, d u) \\
& +\int_{X} \int_{-\infty}^{0}(-u)^{\kappa}\left(G_{1}(x)\left((-u)^{-1} t-1\right)_{+}^{\kappa}+G_{2}(x)\left(\left((-u)^{-1} t-1\right)_{-}^{\kappa}-1\right)\right) \\
& \quad \times M_{\alpha}(d x, d u) \\
& \quad \stackrel{d}{=} \int_{\widetilde{X}} \int_{0}^{\infty} z^{\kappa} \widetilde{G}\left(\tilde{x}, \frac{t}{z}\right) M_{\alpha}(d \tilde{x}, d z),
\end{aligned}
$$

where $\widetilde{X}=X \times\{1,2\}, \widetilde{x}=(x, j, z)$ and $\widetilde{G}(\widetilde{x}, s)=\widetilde{G}(x, j, s)=1_{\{j=1\}} G_{1}(x)((s+$ $\left.1)_{+}^{\kappa}-1\right)+1_{\{j=2\}}\left(G_{1}(x)(s-1)_{+}^{\kappa}+G_{2}(x)\left((s-1)_{-}^{\kappa}-1\right)\right)$ for $x \in X, s>0$ and $j \in\{1,2\}$.

EXAMPLE 5.1. By setting $X=\{1\}$ and $\mu(d x)=\delta_{\{1\}}(d x)$ in (5.1), one gets

$$
X_{\alpha}(t)=\int_{\mathbb{R}}\left(a\left((t+u)_{+}^{\kappa}-u_{+}^{\kappa}\right)+b\left((t+u)_{-}^{\kappa}-u_{-}^{\kappa}\right)\right) M_{\alpha}(d u), \quad t \in \mathbb{R},
$$

that is, the usual linear fractional stable motions. We refer the reader to Chapter 7 in Samorodnitsky and Taqqu (1994) for more information about these processes. Note also that, by setting $X=\{1\}$ and $\mu(d x)=\delta_{\{1\}}(d x)$ in (5.2), one gets

$$
\begin{aligned}
X_{\alpha}(t)=\int_{\mathbb{R}}( & a(\ln |t+u|-\ln |u|) \\
& \left.\quad+b\left(1_{(0, \infty)}(t+u)-1_{(0, \infty)}(u)\right)\right) M_{\alpha}(d u), \quad t \in \mathbb{R} .
\end{aligned}
$$

When $b=0$, the process $(5.12)$ is the so-called log-fractional stable motion introduced by Kasahara, Maejima and Vervaat (1988). If $a=0$, then (5.12) becomes the usual $S \alpha S$ Lévy motion because

$$
X_{\alpha}(t)=b \int_{\mathbb{R}}\left(1_{(0, \infty)}(t+u)-1_{(0, \infty)}(u)\right) M_{\alpha}(d u) \stackrel{d}{=} b \int_{\mathbb{R}} 1_{(0, t)}(u) M_{\alpha}(d u) .
$$

DEFINITION 5.1. We will call processes (5.1) and (5.2) mixed linear fractional stable motions (mixed LFSM, in short).

We give that name to the processes (5.1) because they are extensions of the linear fractional stable motions (5.11). We also call the processes (5.2) mixed LFSM because they can be viewed as limiting cases of mixed LFSM (5.1) when $\kappa \rightarrow 0$. To see this, observe that

$$
\int_{X} \int_{\mathbb{R}}\left(G_{1}(x) \frac{|t+u|^{\kappa}-|u|^{\kappa}}{\kappa}+G_{2}(x)\left((t+u)_{+}^{\kappa}-u_{+}^{\kappa}\right)\right) M_{\alpha}(d x, d u)
$$

is a mixed LFSM, and that $\left(|t+u|^{\kappa}-|u|^{\kappa}\right) / \kappa \rightarrow \ln |t+u|-\ln |u|$ and $(t+u)_{+}^{\kappa}-$ $u_{+}^{\kappa} \rightarrow 1_{(0, \infty)}(t+u)-1_{(0, \infty)}(u)$ as $\kappa \rightarrow 0$.

We conclude this section by applying Theorem 5.1 to characterize linear fractional stable motions, log-fractional stable motion and Lévy motion. This 
characterization extends Theorem 3 in Cambanis, Maejima and Samorodnitsky (1992) because it does not assume the local $L^{1}$-integrability of the kernel function $G$ and also includes the case $H=1 / \alpha$.

Corollary 5.1. Let $\alpha \in(0,2)$ and $H \in(0,1)$. Suppose that a $S \alpha S$ nondegenerate process $\left\{X_{\alpha}(t)\right\}_{t \in \mathbb{R}}$ with stationary increments, having the representation

$$
\left\{X_{\alpha}(t)\right\}_{t \in \mathbb{R}} \stackrel{d}{=}\left\{\int_{\mathbb{R}}(G(t+u)-G(u)) M_{\alpha}(d u)\right\}_{t \in \mathbb{R}},
$$

where $M_{\alpha}$ has the Lebesgue control measure, is self-similar with exponent $H$. Then, if $\kappa=H-1 / \alpha \neq 0, X_{\alpha}$ is a linear fractional stable motion (5.11). If $\kappa=0$, then $X_{\alpha}$ is the sum of the log-fractional stable motion and the Lévy stable motion in (5.12).

Proof. Example 4.1 and Theorem 4.1 in Pipiras and Taqqu (2001a) show that the kernel function $G$ satisfies conditions (2.3) and (2.4). Since $X=\{1\}$, the flow can only be an identity flow. The conclusion then follows from Theorem 5.1.

6. The mixed LFSM component set. According to Theorem 5.1, mixed LFSMs are mixed moving averages $X_{\alpha}$ characterized by identity flows. Another way to get a mixed LFSM is through the kernel $G$ of the process $X_{\alpha}$ directly, without using flows. This idea is based on the mixed LFSM component set which we introduce and whose properties we explore in this section. The set will be used in the next section to decompose the conservative component process $X_{\alpha}^{C}$.

DEFINITION 6.1. Let $X_{\alpha}$ be a mixed moving average (1.4) with the kernel function $G$. The mixed LFSM component set of the process $X_{\alpha}$ is the set

$$
\begin{aligned}
E:= & \left\{x \in X: \exists G_{i}=G_{i}(x), i=1,2,3, g=g(x) \in \mathbb{R}\right. \\
& \text { such that } G(x, u)=G_{1}(x) \ln |u+g(x)| \\
& \left.+G_{2}(x) 1_{(0, \infty)}(u+g(x))+G_{3}(x) \text { a.e. } d u\right\}
\end{aligned}
$$

when $\kappa=0$, and the set

$$
\begin{aligned}
& E:=\left\{x \in X: \exists G_{i}=G_{i}(x), i=1,2,3, g=g(x) \in \mathbb{R}\right. \\
& \text { such that } G(x, u)=G_{1}(x)(u+g(x))_{+}^{\kappa} \\
&\left.+G_{2}(x)(u+g(x))_{-}^{\kappa}+G_{3}(x) \text { a.e. } d u\right\}
\end{aligned}
$$

when $\kappa \neq 0$.

LEMMA 6.1. The mixed LFSM component set E is measurable. The functions $G_{1}, G_{2}, G_{3}$ and $g$, when restricted to $E$ are measurable as well. Moreover, if the function $G$ satisfies condition (2.4), then $\operatorname{supp}\left\{G_{1}, G_{2}\right\}=E$ a.e. 
PROOF. We prove the lemma only in the case $\kappa=0$. The case $\kappa \neq 0$ may be proved in a similar way. The idea is to express this set and the functions $G_{i}$, $i=1,2,3$, and $g$ in terms of the given measurable function $G$. Define first the set

$$
X_{1}=\left\{x \in X: G(x, u)(\ln u)^{-1} \rightarrow \widetilde{G}_{1}(x) \in \mathbb{R} \text { a.e. as } u \rightarrow+\infty\right\},
$$

where by $h(u) \rightarrow h \in \mathbb{R}$ a.e. as $u \rightarrow+\infty$, we mean that $\forall \varepsilon>0 \exists u_{0}=u_{0}(\varepsilon)$ such that $|h(u)-h|<\varepsilon$ a.e. for $u>u_{0}$. We first show that the set $X_{1}$ is measurable. By the Cauchy's criterion, $X_{1}$ is equal to the set $\bigcap_{n \geq 1} \cup_{m \geq 1} A_{n, m}$, where $A_{n, m}=\{x: K(x, u, v)<1 / n$ a.e. $d u d v$ for $u, v>m\}$ and

$$
K(x, u, v)=\left|G(x, u)(\ln u)^{-1}-G(x, v)(\ln v)^{-1}\right| .
$$

Note that the set $A_{n, m}$ is measurable since it equals $\{x: K(x)=0\}$, where the function $K(x)=\int_{\mathbb{R}} \int_{\mathbb{R}} 1_{\{K(x, u, v) \geq 1 / n\}} 1_{\{u, v>m\}} d u d v$ is measurable by the Fubini's theorem. Therefore, $X_{1}$ is measurable. Then, for $x \in X_{1}$, we have

$$
G(x, u+n)(\ln (u+n))^{-1} \rightarrow \widetilde{G}_{1}(x)
$$

for a.e. $u>0$ as $n \rightarrow \infty$, and, by the Fubini's theorem, this convergence holds a.e. for $(x, u) \in X_{1} \times \mathbb{R}$. Since $\widetilde{G}_{1}$ is the limit of measurable functions, it has to be measurable as well. Moreover, we have $E=X_{1} \cap E\left(X_{1}, \widetilde{G}_{1}, G_{2}, G_{3}, g\right)$, where the set $E(\ldots)$ is defined as in (6.1) but with $X$ and $G_{1}$ replaced by $X_{1}$ and $\widetilde{G}_{1}$, respectively, and requiring the existence of $G_{2}, G_{3}$ and $g$ only. [We shall continue to use the $E(\ldots)$ type notation below.] Now, define the set

$$
X_{2}=\left\{x \in X_{1}: G(x, u)-\widetilde{G}_{1}(x) \ln |u| \rightarrow \widetilde{G}_{3}(x) \in \mathbb{R} \text { a.e. as } u \rightarrow-\infty\right\} .
$$

Arguing as above, we can deduce that the set $X_{2}$ is measurable and that the function $\widetilde{G}_{3}$, restricted to $X_{2}$, is measurable as well. Moreover, $E\left(X_{1}, \widetilde{G}_{1}, G_{2}, G_{3}, g\right)=X_{2} \cap E\left(X_{2}, \widetilde{G}_{1}, G_{2}, \widetilde{G}_{3}, g\right)$, since $\ln |u+g(x)|-\ln |u| \rightarrow$ 0 and $1_{(0, \infty)}(u+g(x)) \rightarrow 0$ as $u \rightarrow-\infty$. Next, define the set

$$
\begin{array}{r}
X_{3}=\left\{x \in X_{2}: G(x, u)-\widetilde{G}_{1}(x) \ln |u|-\widetilde{G}_{3}(x) \rightarrow \widetilde{G}_{2}(x) \in \mathbb{R}\right. \\
\text { a.e. as } u \rightarrow+\infty\} .
\end{array}
$$

Again, the set $X_{3}$ is measurable, the function $\widetilde{G}_{2}$, when restricted to $X_{2}$, is measurable as well, and $E\left(X_{2}, \widetilde{G}_{1}, G_{2}, \widetilde{G}_{3}, g\right)=X_{3} \cap E\left(X_{3}, \widetilde{G}_{1}, \widetilde{G}_{2}, \widetilde{G}_{3}, g\right)$. Finally, defining the set

$$
\begin{array}{r}
X_{4}=\left\{x \in X_{3}: \exp \left\{\left(G(x, u)-\widetilde{G}_{3}(x)\right)\left(\widetilde{G}_{1}(x)\right)^{-1} 1_{\left\{\widetilde{G}_{1}(x) \neq 0\right\}}\right\}\right. \\
+u \rightarrow \widetilde{g}(x) \in \mathbb{R} \text { a.e. as } u \rightarrow-\infty\},
\end{array}
$$

we have the measurability of $\widetilde{g}$ and $X_{4}$, and $E\left(X_{3}, \widetilde{G}_{1}, \widetilde{G}_{2}, \widetilde{G}_{3}, g\right)=X_{4} \cap$ $E\left(X_{4}, \widetilde{G}_{1}, \widetilde{G}_{2}, \widetilde{G}_{3}, \widetilde{g}\right)$. Hence, since

$$
E=X_{1} \cap X_{2} \cap X_{3} \cap X_{4} \cap E\left(X_{4}, \widetilde{G}_{1}, \widetilde{G}_{2}, \widetilde{G}_{3}, \widetilde{g}\right),
$$


where each set in the intersection is measurable, we get that $E$ is measurable as well. It is also clear that $G_{i}=\left.\widetilde{G}_{i}\right|_{E}, i=1,2,3$, and $g=\left.\widetilde{g}\right|_{E}$, which implies the measurability of the functions.

If $\operatorname{supp}\left\{G_{1}, G_{2}\right\} \neq E$ a.e., then there is a set $X_{0}$ with $\mu\left(X_{0}\right)>0$ such that $G_{1}(x)=0$ and $G_{2}(x)=0$ for $x \in X_{0}$. Hence, for any $t \in \mathbb{R}, G(x, t+u)-$ $G(x, u)=0$ for $x \in X_{0}$ and $u \in \mathbb{R}$. Since $\mu\left(X_{0}\right)>0$, this contradicts (2.4).

The following lemma shows that the mixed LFSM component set $E$ is a subset of the conservative part of the flow $\left\{\psi_{c}\right\}_{c>0}$.

PROPOSITION 6.1. Suppose that the mixed moving average $X_{\alpha}$ is generated by a flow in the sense of Definition 2.1. Then the mixed LFSM component set $E$ is a subset (a.e.) of the conservative part of the flow $\left\{\psi_{c}\right\}_{c>0}$. Moreover, the set $E$ is invariant under the flow a.e. $d \mu$, that is, for any $c>0, \mu\left(E \triangle \psi_{c}^{-1}(E)\right)=0$.

PROOF. We prove the proposition only for the case $\kappa \neq 0$. The case $\kappa=0$ may be proved in a similar way. By (2.3) and relation (5.11) in Pipiras and Taqqu (2001a), the conservative part of the flow $\left\{\psi_{c}\right\}_{c>0}$ is $C=\{x \in X: I(x)=\infty\}$ a.e. $\mu(d x)$, where

$$
\begin{aligned}
I(x) & =\int_{0}^{\infty} \int_{\mathbb{R}}\left|G\left(\psi_{c}(x), 1+u\right)-G\left(\psi_{c}(x), u\right)\right|^{\alpha} d u \frac{d\left(\mu \circ \psi_{c}\right)}{d \mu}(x) c^{-1} d c \\
& =\int_{0}^{\infty} \int_{\mathbb{R}}|G(x, c(1+u))-G(x, c u)|^{\alpha} d u c^{-H \alpha} d c .
\end{aligned}
$$

The inclusion $E \subset C$ a.e. then follows from (6.2), since, for a.e. $x \in E$,

$$
\begin{aligned}
I(x) & =\int_{0}^{\infty} c^{-1} d c \int_{\mathbb{R}}\left|G_{1}(x)\left((1+u)_{+}^{\kappa}-u_{+}^{\kappa}\right)+G_{2}(x)\left((1+u)_{-}^{\kappa}-u_{-}^{\kappa}\right)\right|^{\alpha} d u \\
& =\infty
\end{aligned}
$$

Let us now show that $\mu\left(E \triangle \psi_{c}^{-1}(E)\right)=0$ for all $c>0$. Fix $c>0$ and consider $x \in E$. By using (2.8) and (6.2), we get for a.e. $\mu(d x)$,

$$
\begin{aligned}
G\left(\psi_{c}(x), u\right) & =\left(b_{c}(x)\right)^{-1}\left\{\frac{d\left(\mu \circ \psi_{c}\right)}{d \mu}\right\}^{1 / \alpha}\left(c^{-\kappa} G\left(x, c\left(u-g_{c}(x)\right)\right)-J(x, c)\right) \\
& =G_{1, c}(x)\left(u+g_{1, c}(x)\right)_{+}^{\kappa}+G_{2, c}(x)\left(u+g_{1, c}(x)\right)_{-}^{\kappa}+G_{3, c}(x)
\end{aligned}
$$

a.e. $d u$, where $G_{1, c}, G_{2, c}, G_{3, c}$ and $g_{1, c}$ are some functions. This shows that $\psi_{c}(x) \in E$ for a.e. $x \in E$ or that $E \subset \psi_{c}^{-1}(E)$ a.e. Since $\psi_{c}^{-1}(E)=\psi_{1 / c}(E)$ and we have that $E \subset \psi_{c}^{-1}(E)$ a.e. implies $\psi_{1 / c}(E) \subset E$ a.e., we get $\psi_{c}^{-1}(E) \subset E$ a.e. and hence $\psi_{c}^{-1}(E)=E$ a.e. 
7. Identification of the mixed LFSM component. We suppose throughout this section that $\alpha \in(1,2)$. Our goal is to show that the conservative part process $X_{\alpha}^{C}$ in (1.7) can be further decomposed uniquely into two independent processes $X_{\alpha}^{(2)}$ and $X_{\alpha}^{(3)}$. The process $X_{\alpha}^{(2)}$ is a mixed LFSM of Definition 5.1, whereas the process $X_{\alpha}^{(3)}$ has no mixed LFSM component, that is, it cannot be decomposed into two independent processes one of which is a mixed LFSM. To obtain our decomposition, we will use the mixed LFSM component set $E$ in Definition 6.1. Another approach, namely that based on the structure of the underlying flow, can be found in Section 10 below.

Let now $X_{\alpha}$ be a $S \alpha S$ self-similar process having representation (1.4) with the kernel function $G$. By Theorem 5.1 in Pipiras and Taqqu (2001a), we may assume without loss of generality that $G$ satisfies the conditions of Definition 2.1 and hence that $X_{\alpha}$ is generated by a multiplicative flow $\left\{\psi_{c}\right\}_{c>0}$ in the sense of that definition. Recall from Pipiras and Taqqu (2001a) that the processes $X_{\alpha}^{D}$ and $X_{\alpha}^{C}$ in the decomposition (1.7) are then defined as

$$
\begin{aligned}
& X_{\alpha}^{D}(t)=\int_{D} \int_{\mathbb{R}}(G(x, t+u)-G(x, u)) M_{\alpha}(d x, d u), \\
& X_{\alpha}^{C}(t)=\int_{C} \int_{\mathbb{R}}(G(x, t+u)-G(x, u)) M_{\alpha}(d x, d u),
\end{aligned}
$$

where $X=D \cup C$ is the Hopf's decomposition of the flow $\left\{\psi_{c}\right\}_{c>0}$ into its dissipative and conservative parts, respectively. Since the mixed LFSM component set $E$ is a subset of the conservative part $C$ by Proposition 6.1, one can decompose the process $X_{\alpha}^{C}$ into two processes as

$$
X_{\alpha}^{C} \stackrel{d}{=} X_{\alpha}^{(2)}+X_{\alpha}^{(3)},
$$

where

$$
\begin{aligned}
& X_{\alpha}^{(2)}(t)=\int_{E} \int_{\mathbb{R}}(G(x, t+u)-G(x, u)) M_{\alpha}(d x, d u), \\
& X_{\alpha}^{(3)}(t)=\int_{C \backslash E} \int_{\mathbb{R}}(G(x, t+u)-G(x, u)) M_{\alpha}(d x, d u) .
\end{aligned}
$$

We will say that the decomposition (7.1) is unique, if it does not depend on the representation (1.4) of $X_{\alpha}$.

PROPOSITION 7.1. The decomposition (7.1) is unique.

Proof. We consider only the case $\kappa \neq 0$. By Theorems 4.2 and 4.1 in Pipiras and Taqqu (2001a), there is a new space $\widetilde{X}$ and function $\widetilde{G}(\tilde{x}, u), \widetilde{x} \in \widetilde{X}, u \in \mathbb{R}$, such that $\{\widetilde{G}(\tilde{x}, t+u)-\widetilde{G}(\tilde{x}, u)\}_{t \in \mathbb{R}} \in L^{\alpha}(\tilde{X} \times \mathbb{R}, \tilde{\mu}(d \tilde{x}) d u)$, which also satisfies the conditions (2.3) and (2.4) in Definition 2.1. This function $\widetilde{G}$ corresponds to the so-called minimal spectral representation of the process $X_{\alpha}$. [For more details, 
see Pipiras and Taqqu (2001a).] Let $\left\{\widetilde{\psi}_{c}\right\}_{c>0}$ be the flow associated to $\widetilde{G}$ by Definition 2.1, $\widetilde{C}$ be the conservative part of the flow $\left\{\widetilde{\psi}_{c}\right\}_{c>0}$, and $\widetilde{E}$ be the set and $\widetilde{G}_{i}, i=1,2,3, \widetilde{g}$ be the functions defined for $\widetilde{G}$ by Definition 6.1. Since $\widetilde{G}$ corresponds to a minimal spectral representation of the process $X_{\alpha}$, by Corollary 5.1 in Pipiras and Taqqu (2001a), there are measurable functions $\Phi_{1}: X \mapsto \widetilde{X}$, $h: X \mapsto \mathbb{R} \backslash\{0\}$ and $\Phi_{2}, \Phi_{3}: X \mapsto \mathbb{R}$ such that

$$
G(x, u)=h(x) \widetilde{G}\left(\Phi_{1}(x), u+\Phi_{2}(x)\right)+\Phi_{3}(x)
$$

a.e. $\mu(d x) d u$ and $\tilde{\mu}=\mu_{h} \circ \Phi_{1}^{-1}$, where $\mu_{h}(d x)=|h(x)|^{\alpha} \mu(d x)$. We want to show first that $\Phi_{1}^{-1}(\widetilde{E})=E$ and that $\Phi_{1}^{-1}(\widetilde{C} \backslash \widetilde{E})=C \backslash E$ a.e. $\mu(d x)$. If $x \in E$, then a.e. $\mu(d x)$,

$$
\begin{gathered}
G_{1}(x)(u+g(x))_{+}^{\kappa}+G_{2}(x)(u+g(x))_{-}^{\kappa}+G_{3}(x) \\
=h(x) \widetilde{G}\left(\Phi_{1}(x), u+\Phi_{2}(x)\right)+\Phi_{3}(x)
\end{gathered}
$$

or

$$
\begin{aligned}
\widetilde{G}\left(\Phi_{1}(x), u\right)= & \frac{G_{1}(x)}{h(x)}\left(u-\Phi_{2}(x)+g(x)\right)_{+}^{\kappa} \\
& +\frac{G_{2}(x)}{h(x)}\left(u-\Phi_{2}(x)+g(x)\right)_{-}^{\kappa}+\frac{G_{3}(x)-\Phi_{3}(x)}{h(x)}
\end{aligned}
$$

a.e. $d u$, which shows that $\Phi_{1}(x) \in \widetilde{E}$ and hence $E \subset \Phi_{1}^{-1}(\widetilde{E})$ a.e. $\mu(d x)$. Conversely, if $\Phi_{1}(x) \in \widetilde{E}$, then a.e. $\mu(d x)$,

$$
\begin{aligned}
G(x, u)=h(x) & \left(\widetilde{G}_{1}(x)\left(u+\Phi_{2}(x)+\widetilde{g}(x)\right)_{+}^{\kappa}\right. \\
& \left.+\widetilde{G}_{2}(x)\left(u+\Phi_{2}(x)+\widetilde{g}(x)\right)_{-}^{\kappa}+\widetilde{G}_{3}(x)\right)+\Phi_{3}(x)
\end{aligned}
$$

a.e. $d u$, which, by performing multiplications on the right-hand side, implies $x \in E$ and hence $\Phi_{1}^{-1}(\widetilde{E}) \subset E$ a.e. $\mu(d x)$. Therefore, $\Phi_{1}^{-1}(\widetilde{E})=E$ a.e. $\mu(d x)$. Relation (5.17) in the proof of Theorem 5.3 in Pipiras and Taqqu (2001a) shows that $\Phi_{1}^{-1}\left(\widetilde{C}_{0}\right)=C_{0}$ a.e. $\mu(d x)$, where $C_{0}=C$ a.e. $\mu(d x)$ and $\widetilde{C}_{0}=\widetilde{C}$ a.e. $\tilde{\mu}(d \widetilde{x})$, and hence $\Phi_{1}^{-1}(\widetilde{C})=C$ a.e. $\mu(d x)$, since by using $\tilde{\mu}=\mu_{h} \circ \Phi_{1}^{-1}, \tilde{\mu}(\tilde{N})=0$ for $\tilde{N} \in \tilde{X}$ implies $\mu\left(\Phi^{-1}(\tilde{N})\right)=0$. Together with $\Phi_{1}^{-1}(\widetilde{E})=E$ a.e. $\mu(d x)$, this implies that $\Phi_{1}^{-1}(\widetilde{C} \backslash \widetilde{E})=C \backslash E$ a.e. $\mu(d x)$.

Let now $\widetilde{X}_{\alpha}^{(2)}$ and $\widetilde{X}_{\alpha}^{(3)}$ be the processes in the decomposition (7.1) obtained from replacing $G, E, C$ by $\widetilde{G}, \widetilde{E}, \widetilde{C}$ in (7.2) and (7.3), respectively. Then, for 
every $a_{1}, \ldots, a_{n} \in \mathbb{R}$ and $t_{1}, \ldots, t_{n} \in \mathbb{R}, n \geq 1$, we have, by (7.4),

$$
\begin{aligned}
-\ln & E \exp \left\{i \sum_{k=1}^{n} a_{k} X_{\alpha}^{(2)}\left(t_{k}\right)\right\} \\
& =\int_{E} \int_{\mathbb{R}}\left|\sum_{k=1}^{n} a_{k}\left(G\left(x, t_{k}+u\right)-G(x, u)\right)\right|^{\alpha} \mu(d x) d u \\
& =\int_{E} \int_{\mathbb{R}}\left|\sum_{k=1}^{n} a_{k}\left(\widetilde{G}\left(\Phi_{1}(x), t_{k}+u+\Phi_{2}(x)\right)-\widetilde{G}\left(\Phi_{1}(x), u+\Phi_{2}(x)\right)\right)\right|^{\alpha} \\
& =\int_{\Phi_{1}^{-1}(\widetilde{E})} \int_{\mathbb{R}}\left|\sum_{k=1}^{n} a_{k}\left(\widetilde{G}\left(\Phi_{1}(x), t_{k}+u\right)-\widetilde{G}\left(\Phi_{1}(x), u\right)\right)\right|^{\alpha} \mu_{h}(d x) d u \\
= & \int_{\widetilde{E}} \int_{\mathbb{R}}\left|\sum_{k=1}^{n} a_{k}\left(\widetilde{G}\left(\widetilde{x}, t_{k}+u\right)-\widetilde{G}(\tilde{x}, u)\right)\right|^{\alpha} \widetilde{\mu}(d \widetilde{x}) d u \\
= & -\ln E \exp \left\{i \sum_{k=1}^{n} a_{k} \widetilde{X}_{\alpha}^{(2)}\left(t_{k}\right)\right\}
\end{aligned}
$$

which shows that $X_{\alpha}^{(2)}={ }_{d} \tilde{X}_{\alpha}^{(2)}$. Similarly, $X_{\alpha}^{(3)}={ }_{d} \tilde{X}_{\alpha}^{(3)}$ and hence decomposition (7.1) is unique.

We will say that a process does not have a mixed LFSM component, if it cannot be decomposed into two independent $S \alpha S$ processes one of which is a mixed LFSM. We will also say that two processes $X$ and $\widetilde{X}$ are essentially different if there is no multiplicative constant $c$ such that $X(t)$ and $c \tilde{X}(t)$ have the same finitedimensional distributions.

Proposition 7.2. The process $X_{\alpha}^{(2)}$ in (7.2) is a mixed LFSM, while the process $X_{\alpha}^{(3)}$ in (7.3) is a self-similar process with stationary increments, generated by a conservative flow, which does not have a mixed LFSM component. These processes are independent and essentially different.

PROOF. The processes $X_{\alpha}^{(2)}$ and $X_{\alpha}^{(3)}$ are independent because their kernels have disjoint supports, that is, $E \cap(C \backslash E)=\varnothing$ [see Theorem 3.5.3 in Samorodnitsky and Taqqu (1994)]. The process $X_{\alpha}^{(2)}$ is a mixed LFSM by construction. The process $X_{\alpha}^{(3)}$ has representation (1.4) and hence has stationary increments. It is also self-similar. Indeed, $X_{\alpha}^{C}={ }_{d} X_{\alpha}^{(2)}+X_{\alpha}^{(3)}, X_{\alpha}^{(2)}$ and $X_{\alpha}^{(3)}$ 
are independent and $X_{\alpha}^{C}$ and $X_{\alpha}^{(2)}$ are self-similar. Therefore, $X_{\alpha}^{(3)}$ is self-similar because its characteristic function is the ratio of the characteristic functions of two self-similar processes. Let us show now that $X_{\alpha}^{(3)}$ has no mixed LFSM component when $\kappa \neq 0$. Suppose there exist two independent $S \alpha S$ processes $Y_{\alpha}$ and $Z_{\alpha}$ such that

$$
X_{\alpha}^{(3)} \stackrel{d}{=} Y_{\alpha}+Z_{\alpha}
$$

where $Y_{\alpha}$ is a mixed LFSM, defined by (5.1) with some functions $\widehat{G}_{1}, \widehat{G}_{2} \subset$ $L^{\alpha}(Y, \widehat{\mu}(d y))$ and $\operatorname{supp}\left\{\widehat{G}_{1}, \widehat{G}_{2}\right\}=Y$. The structure of the process $Z_{\alpha}$ plays no role in the proof. Observe only that, by (7.5), $Z_{\alpha}$ has a stochastic integral representation. Indeed, since $S \alpha S$ processes $Y_{\alpha}+Z_{\alpha}\left(={ }_{d} X_{\alpha}^{(3)}\right)$ and $-Y_{\alpha}$ have stochastic integral representations, these processes are necessarily separable in probability and hence so is their sum $Z_{\alpha}$ [see Theorem 13.2.1 and Exercise 3.20 in Samorodnitsky and Taqqu (1994)]. Then, by the same Theorem 13.2.1, we have $Z_{\alpha}(t)={ }_{d} \int_{0}^{1} g_{t}(z) M_{\alpha}(d z)$ for some $\left\{g_{t}\right\}_{t \in \mathbb{R}} \subset L^{\alpha}(0,1)$, where $M_{\alpha}$ has the Lebesgue control measure $d z$. Since $Y_{\alpha}$ and $Z_{\alpha}$ are independent, one can represent the sum $Y_{\alpha}+Z_{\alpha}$ as $\int_{V} f_{t}(v) M_{\alpha}(d v)$, where $V$ is made up of two disjoint parts $Y \times \mathbb{R}$ and $(0,1)$. Formally, $V=(Y \times \mathbb{R}) \cup(0,1)=\{v: v=(y, u) \in$ $Y \times \mathbb{R}$ or $v=z \in(0,1)\}, f_{t}(v)=g_{t}(z)$, if $v=z$, and $f_{t}(v)=\widehat{G}_{1}(y)\left((t+u)_{+}^{\kappa}-\right.$ $\left.u_{+}^{\kappa}\right)+\widehat{G}_{2}(y)\left((t+u)_{-}^{\kappa}-u_{-}^{\kappa}\right)$, if $v=(y, u)$, and $M_{\alpha}$ has the control measure $m(d v)$ equal to $d z$ on $(0,1)$ and $\widehat{\mu}(d y) d u$ on $Y \times \mathbb{R}$. We may suppose without loss of generality that $\operatorname{supp}\left\{f_{t}, t \in \mathbb{R}\right\}=V$. Then, one can relate the representations of $Y_{\alpha}+Z_{\alpha}$ and $X_{\alpha}^{(3)}$ in (7.5): by Theorem 1.1 in Rosiński (1995), there are functions $\Phi_{1}: V \mapsto C \backslash E, \Phi_{2}: V \mapsto \mathbb{R}$ and $h: V \mapsto \mathbb{R} \backslash\{0\}$ such that

$$
f_{t}(v)=h(v)\left(G\left(\Phi_{1}(v), t+\Phi_{2}(v)\right)-G\left(\Phi_{1}(v), \Phi_{2}(v)\right)\right)
$$

a.e. $m(d v) d t$. Considering only those $v=(y, u) \in Y \times \mathbb{R}$, we have

$$
\begin{aligned}
& \widehat{G}_{1}(y)\left((t+u)_{+}^{\kappa}-u_{+}^{\kappa}\right)+\widehat{G}_{2}(y)\left((t+u)_{-}^{\kappa}-u_{-}^{\kappa}\right) \\
& \quad=h(y, u)\left(G\left(\Phi_{1}(y, u), t+\Phi_{2}(y, u)\right)-G\left(\Phi_{1}(y, u), \Phi_{2}(y, u)\right)\right)
\end{aligned}
$$

a.e. $\widehat{\mu}(d y) d u d t$. Then fixing $u=u_{0}$ for which the relation holds a.e. $\widehat{\mu}(d y) d t$ and making the change of variables $t+u_{0}=w$, we get that there are new measurable functions $\Phi_{1}: Y \mapsto C \backslash E, \Phi_{2}, \Phi_{3}: Y \mapsto \mathbb{R}$ and $h: Y \mapsto \mathbb{R} \backslash\{0\}$ such that

$$
\widehat{G}_{1}(y) w_{+}^{\kappa}+\widehat{G}_{1}(y) w_{-}^{\kappa}=h(y) G\left(\Phi_{1}(y), w+\Phi_{2}(y)\right)+\Phi_{3}(y)
$$

a.e. $\widehat{\mu}(d y) d w$. Hence, $\Phi_{1}(y) \in E$ a.e. $\widehat{\mu}(d y)$. If $\widehat{\mu}$ is not a zero measure, then relation (7.6) contradicts the fact that $\Phi_{1}(y) \in C \backslash E$. The case of $\kappa=0$ may be proved in a similar way.

Finally, there is no constant $c \neq 0$ such that $c X_{\alpha}^{(2)}={ }_{d} X_{\alpha}^{(3)}$ (in other words, the processes are essentially different), since $X_{\alpha}^{(3)}$ has no mixed LFSM component. 
8. Example of a process of the "third kind." In this section, we provide an example of a mixed moving average process generated by a conservative flow which has only process $X_{\alpha}^{(3)}$ in its decomposition (7.1). (We refer to such processes as processes of the "third kind.") Existence of such a process turned out to be a nontrivial problem.

Consider a mixed moving average $X_{\alpha}$ in (1.4) with $X=[0,1), \mu(d x)=d x$ and

$$
G(x, u)=u_{+}^{\kappa} 1_{[0,1 / 2)}(\{x+\ln |u|\}), \quad x \in[0,1), u \in \mathbb{R},
$$

where $\kappa=H-1 / \alpha<0, H \in(0,1), \alpha \in(0,2)$ and $\{\cdot\}$ denotes the fractional part function. By using Lemma 8.1 below, the process $X_{\alpha}$ is well-defined for $\kappa<0$ and thus it can be represented as

$$
\begin{aligned}
X_{\alpha}(t)=\int_{0}^{1} \int_{\mathbb{R}}( & \left.(t+u)_{+}^{\kappa} 1_{[0,1 / 2)}(\{x+\ln |t+u|\})-u_{+}^{\kappa} 1_{[0,1 / 2)}(\{x+\ln |u|\})\right) \\
& \times M_{\alpha}(d x, d u),
\end{aligned}
$$

where $S \alpha S$ random measure $M_{\alpha}$ has the Lebesgue control measure on $[0,1) \times \mathbb{R}$. By using the identity

$$
\{x+s+t\}=\{\{x+s\}+t\}, \quad x \in[0,1), s, t \in \mathbb{R},
$$

we obtain from (8.1) that, for all $c>0$ and $x \in[0,1)$,

$$
c^{-\kappa} G(x, c u)=u_{+}^{\kappa} 1_{[0,1 / 2)}(\{\{x+\ln c\}+\ln |u|\})=G\left(\psi_{c}(x), u\right),
$$

where $\psi_{c}(x)=\{x+\ln c\}$.

The identity (8.3) also shows that $\left\{\psi_{c}\right\}_{c>0}$ is a multiplicative flow on $[0,1)$. We will call the multiplicative flow $\left\{\psi_{c}\right\}_{c>0}$ a cycle because its additive counterpart, the flow $\phi_{t}(x)=\psi_{e^{t}}(x)=\{x+t\}, t \in \mathbb{R}$, is isomorphic to a cycle $\widetilde{\phi}_{t}(z)=e^{i 2 \pi t} z$, $t \in \mathbb{R}$, on the unit circle $|z|=1$. Observe that a cycle is clearly conservative since the flow is measure preserving and given a set $A \subset[0,1)$ with $\mu(A)>0$, the iterations of $A$ under the flow cannot be disjoint since they are in $[0,1)$ and $\mu[0,1)=1<\infty$. Relation (8.4) and Definition 2.1 now imply that the process (8.2) is generated by a conservative flow, namely a cycle $\left\{\psi_{c}\right\}_{c>0}$, and by the corresponding cocycle $b_{c}(x) \equiv 0$ and the semi-additive functional $g_{c}(x) \equiv 0$. Since the mixed LFSM component set $E$ for the kernel function (8.1) is empty, the process (8.2) is indeed a process of the "third kind," that is, it is of the type $X_{\alpha}^{(3)}$.

In the next lemma, we prove that the process (8.2) is well defined for $\kappa<0$.

Lemma 8.1. Let $\alpha \in(0,2), H \in(0,1)$ and $\kappa=H-1 / \alpha<0$. Set $G_{t}(x, u)=$ $G(x, t+u)-G(x, u)$ for $x \in[0,1)$ and $u, t \in \mathbb{R}$, where the function $G$ is defined by (8.1). Then $G_{t} \in L^{\alpha}([0,1) \times \mathbb{R})$ for each $t \in \mathbb{R}$. 
PROOF. Since $\left\{G_{t}\right\}_{t \in \mathbb{R}}$ is the kernel of a self-similar process $X_{\alpha}$, it is enough to prove that $G_{1} \in L^{\alpha}([0,1) \times \mathbb{R})$, that is,

$$
\begin{aligned}
\int_{0}^{1} \int_{\mathbb{R}} \mid(1+u)_{+}^{\kappa} 1_{[0,1 / 2)}(\{x+\ln |1+u|\}) \\
-\left.u_{+}^{\kappa} 1_{[0,1 / 2)}(\{x+\ln |u|\})\right|^{\alpha} d x d u<\infty
\end{aligned}
$$

or, with $B=\bigcup_{k \in \mathbb{Z}}[k, k+1 / 2)$,

$$
\int_{0}^{1} \int_{\mathbb{R}}\left|(1+u)_{+}^{\kappa} 1_{\{(x+\ln |1+u|) \in B\}}-u_{+}^{\kappa} 1_{\{(x+\ln |u|) \in B\}}\right|^{\alpha} d x d u<\infty .
$$

It is also enough to verify that

$$
J=\int_{0}^{1} \int_{13}^{\infty}\left|(1+u)^{\kappa} 1_{\{(x+\ln (1+u)) \in B\}}-u^{\kappa} 1_{\{(x+\ln u) \in B\}}\right|^{\alpha} d x d u<\infty
$$

(the lower limit is 13 for good luck), since $\kappa \alpha+1=H \alpha>0$ and, for example, $\int_{-1}^{13}|1+u|^{\kappa \alpha} d u<\infty$. Observe now that, for $u>0$,

$$
1_{\{(x+\ln (1+u)) \in B\}}=1_{\left\{u \in \cup_{k \in \mathbb{Z}}\left[e^{k-x}-1, e^{k-x+1 / 2}-1\right)\right\}}
$$

and

$$
1_{\{(x+\ln u) \in B\}}=1_{\left\{u \in \cup_{k \in \mathbb{Z}}\left[e^{k-x}, e^{k-x+1 / 2}\right)\right\}} .
$$

Suppose $k \geq 3$ and $x \in[0,1)$. Since $e^{k-x}<e^{k-x+1 / 2}-1$, we have $e^{k-x}-1 \leq$ $e^{k-x} \leq e^{k-x+1 / 2}-1 \leq e^{k-x+1 / 2}$. Moreover, since $e^{(k-1)-x+1 / 2}<e^{k-x}-1$, the intervals $\left(e^{k-x}-1, e^{k-x+1 / 2}\right]$ do not overlap. Then, since $e^{(k-1)-x+1 / 2}<13$ for $k \leq 3$ and all $x \in[0,1)$, we obtain that

$$
\begin{aligned}
& J=\int_{0}^{1} \int_{13}^{\infty} \mid \sum_{k=-\infty}^{\infty}(1+u)^{\kappa} 1_{\left\{u \in \cup_{k \in \mathbb{Z}}\left[e^{k-x}-1, e^{k-x+1 / 2}-1\right)\right\}} \\
& -\left.u^{\kappa} 1_{\left\{u \in \cup_{k \in \mathbb{Z}}\left[e^{k-x}, e^{k-x+1 / 2}\right)\right\}}\right|^{\alpha} d x d u \\
& \leq \int_{0}^{1} \int_{0}^{\infty} \mid \sum_{k=3}^{\infty}(1+u)^{\kappa} 1_{\left\{u \in \cup_{k \in \mathbb{Z}}\left[e^{k-x}-1, e^{k-x+1 / 2}-1\right)\right\}} \\
& -\left.u^{\kappa} 1_{\left\{u \in \cup_{k \in \mathbb{Z}}\left[e^{k-x}, e^{k-x+1 / 2}\right)\right\}}\right|^{\alpha} d x d u \\
& \leq \int_{0}^{1} \int_{0}^{\infty} \mid \sum_{k=3}^{\infty}(1+u)^{\kappa} 1_{\left\{u \in\left[e^{k-x}-1, e^{k-x}\right)\right\}}+\left((1+u)^{\kappa}-u^{\kappa}\right) \\
& \times 1_{\left\{u \in\left[e^{k-x}, e^{k-x+1 / 2}-1\right)\right\}}+\left.u^{\kappa} 1_{\left\{u \in\left[e^{k-x+1 / 2}-1, e^{k-x+1 / 2}\right)\right\}}\right|^{\alpha} d x d u
\end{aligned}
$$




$$
\begin{aligned}
\leq & 3^{\alpha} \int_{0}^{1} \int_{0}^{\infty}\left|\sum_{k=3}^{\infty}(1+u)^{\kappa} 1_{\left\{u \in\left[e^{k-x}-1, e^{k-x}\right)\right\}}\right|^{\alpha} d x d u \\
& +3^{\alpha} \int_{0}^{1} \int_{0}^{\infty}\left|(1+u)^{\kappa}-u^{\kappa}\right|^{\alpha} d x d u \\
& +3^{\alpha} \int_{0}^{1} \int_{0}^{\infty}\left|\sum_{k=3}^{\infty} u^{\kappa} 1_{\left\{u \in\left[e^{k-x+1 / 2}-1, e^{k-x+1 / 2}\right)\right\}}\right|^{\alpha} d x d u \\
\leq & 3^{\alpha} \int_{0}^{1} \sum_{k=3}^{\infty} e^{(k-x) \kappa \alpha} d x \\
& +3^{\alpha} \int_{0}^{\infty}\left|(1+u)^{\kappa}-u^{\kappa}\right|^{\alpha} d u+3^{\alpha} \int_{0}^{1} \sum_{k=3}^{\infty}\left(e^{k-x+1 / 2}-1\right)^{\kappa \alpha} d x<\infty,
\end{aligned}
$$

since $|a+b+c|^{\alpha} \leq 3^{\alpha} \max \left\{|a|^{\alpha},|b|^{\alpha},|c|^{\alpha}\right\} \leq 3^{\alpha}\left(|a|^{\alpha}+|b|^{\alpha}+|c|^{\alpha}\right), \kappa<0$, $\int_{0}^{\infty}\left|(1+u)^{\kappa}-u^{\kappa}\right|^{\alpha} d u<\infty$ for $\alpha \in(0,2)$ and $H \in(0,1)$, and $\sum_{k=3}^{\infty} e^{k \kappa \alpha}=$ $\sum_{k=3}^{\infty} e^{-k|\kappa| \alpha}<\infty$ for $\kappa<0$.

9. Decomposition of the process in three components. The following theorem summarizes the preceding results.

TheOrem 9.1. Let $\alpha \in(1,2), H \in(0,1)$ and $\kappa=H-1 / \alpha$. Suppose that the process $X_{\alpha}$ has representation (1.4) (and hence has stationary increments) and is self-similar with exponent $H$. Then, it can be uniquely decomposed in distribution into three independent processes

$$
X_{\alpha} \stackrel{d}{=} X_{\alpha}^{(1)}+X_{\alpha}^{(2)}+X_{\alpha}^{(3)} .
$$

The process $X_{\alpha}^{(1)}$ has the canonical representation (4.1) and is generated by a dissipative multiplicative flow in the sense of Definition 2.1. The process $X_{\alpha}^{(2)}$, called a mixed linear fractional stable motion, is generated by a conservative flow in the sense of Definition 2.1 and has the representation (5.1), if $\kappa \neq 0$, and (5.2), if $\kappa=0$. The process $X_{\alpha}^{(3)}$ is also generated by a conservative flow in the sense of Definition 2.1, but has no mixed linear fractional stable motion component.

We obtained the decomposition (9.1), by assuming, without loss of generality, that the self-similar stationary increments mixed moving average $X_{\alpha}$ is generated by a flow $\left\{\psi_{c}\right\}_{c>0}$ associated with a kernel $G$ as in Definition 2.1. An alternative way to obtain (9.1) is as follows. Suppose that $X_{\alpha}$ is an $H$-self-similar process having the representation (1.4) and that condition (2.4) holds [without assuming (2.3)]. Setting

$$
I(x)=\int_{0}^{\infty} c^{-H \alpha} \int_{\mathbb{R}}|G(x, c(1+u))-G(x, c u)|^{\alpha} d u d c
$$


for $x \in X$, define the sets

$$
D=\{x: I(x)<\infty\} \text { and } C=\{x: I(x)=\infty\} .
$$

Even though the sets $D$ and $C$ are not associated with a flow, one can obtain a decomposition of the type (9.1), by defining first the processes

$$
X_{\alpha}^{D}(t)=\int_{D} \int_{\mathbb{R}}(G(x, t+u)-G(x, u)) M_{\alpha}(d x, d u)
$$

and

$$
X_{\alpha}^{C}(t)=\int_{C} \int_{\mathbb{R}}(G(x, t+u)-G(x, u)) M_{\alpha}(d x, d u),
$$

and then decomposing $X_{\alpha}^{C}$ further into two independent processes

$$
X_{\alpha}^{E}(t)=\int_{E} \int_{\mathbb{R}}(G(x, t+u)-G(x, u)) M_{\alpha}(d x, d u)
$$

and

$$
X_{\alpha}^{C \backslash E}(t)=\int_{C \backslash E} \int_{\mathbb{R}}(G(x, t+u)-G(x, u)) M_{\alpha}(d x, d u),
$$

where $E$ is the set defined in Definition 6.1. The proof of Proposition 6.1 shows that $E$ is indeed a subset of $C$ a.e. $\mu(d x)$ [where $C$ is now defined by (9.3)]. The following result states that the processes $X_{\alpha}^{D}, X_{\alpha}^{E}$ and $X_{\alpha}^{C \backslash E}$ are equal in distribution to the three components in the decomposition (9.1).

Corollary 9.1. Let $\alpha \in(1,2)$ and $H \in(0,1)$. Suppose that the $H$-selfsimilar process $X_{\alpha}$ has representation (1.4) with $\operatorname{supp}\{G(x, t+u)-G(x, u)$, $t \in \mathbb{R}\}=X \times \mathbb{R}$. Let $X_{\alpha}^{D}, X_{\alpha}^{E}$ and $X_{\alpha}^{C \backslash E}$ be the processes defined in (9.4), (9.6) and (9.7) by using the sets $D, C$ and $E$ in (9.3) and Definition 6.1, respectively. Then

$$
X_{\alpha}^{D} \stackrel{d}{=} X_{\alpha}^{(1)}, \quad X_{\alpha}^{E} \stackrel{d}{=} X_{\alpha}^{(2)}, \quad X_{\alpha}^{C \backslash E} \stackrel{d}{=} X_{\alpha}^{(3)},
$$

where the processes $X_{\alpha}^{(1)}, X_{\alpha}^{(2)}$ and $X_{\alpha}^{(3)}$ are the three components in the decomposition (9.1).

Proof. By Corollary 5.3 in Pipiras and Taqqu (2001a), the processes $X_{\alpha}^{D}$ and $X_{\alpha}^{C}$ defined by (9.4) and (9.5) are (in distribution) the dissipative and conservative components in the decomposition (1.7) of $X_{\alpha}$. In particular, $X_{\alpha}^{D}={ }_{d} X_{\alpha}^{(1)}$, where $X_{\alpha}^{(1)}$ is the first process in the decomposition (9.1). As in the proof of Proposition 7.1 [see also relation (5.25) in Pipiras and Taqqu (2001a)], one can show that $X_{\alpha}^{E}={ }_{d} \tilde{X}_{\alpha}^{(2)}$ and $X_{\alpha}^{C \backslash E}={ }_{d} \widetilde{X}_{\alpha}^{(3)}$, where the processes $\widetilde{X}_{\alpha}^{(2)}$ and $\widetilde{X}_{\alpha}^{(3)}$ are defined in the proof of that proposition. Since, by the same proposition, the 
decomposition (7.1) is unique, we obtain $X_{\alpha}^{E}={ }_{d} X_{\alpha}^{(2)}$ and $X_{\alpha}^{C \backslash E}={ }_{d} X_{\alpha}^{(3)}$, where $X_{\alpha}^{(2)}$ and $X_{\alpha}^{(3)}$ are the last two processes in the decomposition (9.1).

Corollary 9.1 is useful because it provides a simple expression for $D$ and $C$ which only involves the representation (1.4) of the process.

10. Connection between decomposition and flows. Let $\alpha \in(1,2)$ and $X_{\alpha}$ be a self-similar mixed moving average (1.4) with the kernel function $G$. As in Section 7, we may suppose without loss of generality that $X_{\alpha}$ is generated by a flow $\left\{\psi_{c}\right\}_{c>0}$ associated with the kernel $G$ as in Definition 2.1. Recall from Pipiras and Taqqu (2001a) that $X_{\alpha}$ can be uniquely decomposed as $X_{\alpha}={ }_{d} X_{\alpha}^{D}+X_{\alpha}^{C}$, where $X_{\alpha}^{D}$ is generated by a dissipative component $\left\{\psi_{c}^{D}\right\}_{c>0}$ of the flow $\left\{\psi_{c}\right\}_{c>0}$ and $X_{\alpha}^{C}$ is generated by a conservative component $\left\{\psi_{c}^{C}\right\}_{c>0}$ of the flow $\left\{\psi_{c}\right\}_{c>0}$. We saw in Section 7 that one can decompose $X_{\alpha}^{C}$ further in a unique way as $X_{\alpha}^{C}={ }_{d} X_{\alpha}^{(2)}+X_{\alpha}^{(3)}$, where $X_{\alpha}^{(2)}$ is a mixed LFSM and $X_{\alpha}^{(3)}$ does not have a mixed LFSM component. Unlike the decomposition of $X_{\alpha}$ into the dissipative part $X_{\alpha}^{D}$ and the conservative part $X_{\alpha}^{C}$, the decomposition of $X_{\alpha}^{C}$ into a mixed LFSM $X_{\alpha}^{(2)}$ and a "third kind" process $X_{\alpha}^{(3)}$ is not related directly to the flow but to the kernel $G$ itself (through the set $E$ in Definition 6.1). The question then is when and how the processes $X_{\alpha}^{(2)}$ and $X_{\alpha}^{(3)}$ can be associated with the underlying flow $\left\{\psi_{c}\right\}_{c>0}$.

A natural candidate for this connection is the set of the "fixed points" of the flow $\left\{\psi_{c}\right\}_{c>0}$, namely,

$$
F=\left\{x \in X: \psi_{c}(x)=x \text { for all } c>0\right\},
$$

which is a subset of a conservative part $C$ of the flow. Since, by Theorem 5.1, the process

$$
X_{\alpha}^{F}(t)=\int_{F} \int_{\mathbb{R}}(G(x, t+u)-G(x, u)) M_{\alpha}(d x, d u)
$$

has a mixed LFSM representation, one may expect that $X_{\alpha}^{F}={ }_{d} X_{\alpha}^{(2)}$ and, similarly, $X_{\alpha}^{C \backslash F}={ }_{d} X_{\alpha}^{(3)}$, where

$$
X_{\alpha}^{C \backslash F}(t)=\int_{C \backslash F} \int_{\mathbb{R}}(G(x, t+u)-G(x, u)) M_{\alpha}(d x, d u) .
$$

This is, however, incorrect in general. For example, the mixed LFSM

$$
X_{\alpha}(t)=\int_{0}^{1} \int_{\mathbb{R}}\left((t+u)_{+}^{\kappa}-u_{+}^{\kappa}\right) M_{\alpha}(d x, d u),
$$

where $M_{\alpha}$ has the Lebesgue control measure on $[0,1) \times \mathbb{R}$, can be generated by a cycle $\psi_{c}(x)=\{x+\ln c\}, x \in[0,1), c>0$ (see Section 8 , where the notion of a cycle is introduced) and hence $F=\varnothing$ and $C \backslash F=[0,1)$. Therefore, in this case, 
$X_{\alpha}^{F} \equiv 0$ and $X_{\alpha}^{C \backslash F}=X_{\alpha}$ while, by (7.2) and (7.3), $X_{\alpha}^{(2)}=X_{\alpha}$ and $X_{\alpha}^{(3)} \equiv 0$ and hence $X_{\alpha}^{(2)} \neq_{d} X_{\alpha}^{F}$.

We will show here, however, that, if the representation (1.4) is minimal for the process $X_{\alpha}$, then $X_{\alpha}^{(2)}$ is indeed the process $X_{\alpha}^{F}$ in (10.2) and, similarly, $X_{\alpha}^{(3)}={ }_{d} X_{\alpha}^{C \backslash F}$. Minimal representations are defined and discussed at length in Pipiras and Taqqu (2001a). They are natural in our context because they rule out "redundancies", as in the representation (10.4). [Note that the variable $x$ in (10.4) is not doing much.] From another perspective, we may say that, when one considers minimal representations, the process $X_{\alpha}^{(2)}$ in (7.2) is generated by the identity flow only. The following two results make this precise.

THEOREM 10.1. Let $\alpha \in(1,2)$. If the representation (1.4) is minimal for a self-similar process $X_{\alpha}$ and a flow $\left\{\psi_{c}\right\}_{c>0}$ is associated with this representation as in Definition 2.1, then the set $E$ in Definition 6.1 satisfies

$$
E=\left\{x \in X: \psi_{c}(x)=x \text { for all } c>0\right\} \quad \text { a.e. } d \mu .
$$

Hence the corresponding process $X_{\alpha}^{(2)}$ is generated by the identity flow only.

PROOF. We shall give the proof for $\kappa \neq 0$. The case $\kappa=0$ is proved in a similar way. In view of Proposition 6.1, the set $E$ is invariant under the flow a.e. $d \mu$. By Lemma 2.3 in Krengel (1969), there is a set $E^{*}$ such that $E=E^{*}$ a.e. $\mu(d x)$ and $E^{*}$ is $\left\{\psi_{c}\right\}$-invariant, that is, $\psi_{c}\left(E^{*}\right)=E^{*}$ for all $c>0$. Since $E=E^{*}$ a.e. $\mu(d x)$, replacing $E$ by $E^{*}$ in the representation (7.2) of $X_{\alpha}^{(2)}$ does not change the distribution of the process, that is,

$$
\left\{X_{\alpha}^{(2)}(t)\right\}_{t \in \mathbb{R}} \stackrel{d}{=}\left\{\int_{E^{*}} \int_{\mathbb{R}}(G(x, t+u)-G(x, u)) M_{\alpha}(d x, d u)\right\}_{t \in \mathbb{R}} .
$$

Since $E^{*}$ is a $\left\{\psi_{c}\right\}$-invariant set, the collection of maps $\left\{\psi_{c}^{*}\right\}_{c>0}=\left\{\left.\psi_{c}\right|_{E^{*}}\right\}_{c>0}$ is a flow on $E^{*}$, and $\left\{b_{c}^{*}\right\}_{c>0}=\left\{\left.b_{c}\right|_{E^{*}}\right\}_{c>0}$ and $\left\{g_{c}^{*}\right\}_{c>0}=\left\{\left.g_{c}\right|_{E^{*}}\right\}_{c>0}$ are a cocycle and a semi-additive functional for the flow $\left\{\psi_{c}^{*}\right\}_{c>0}$. Then, in view of Definition 2.1, we have for $c>0$,

$$
\begin{aligned}
c^{-\kappa}( & G(x, c(t+u))-G(x, c u)) \\
= & b_{c}^{*}(x)\left\{\frac{d\left(\mu \circ \psi_{c}^{*}\right)}{d \mu}(x)\right\}^{1 / \alpha} \\
& \times\left(G\left(\psi_{c}^{*}(x), t+u+g_{c}^{*}(x)\right)-G\left(\psi_{c}^{*}(x), u+g_{c}^{*}(x)\right)\right)
\end{aligned}
$$

a.e. for $(x, u) \in E^{*} \times \mathbb{R}$, and hence the process $X_{\alpha}^{(2)}$ is generated by the flow $\left\{\psi_{c}^{*}\right\}_{c>0}$. On the other hand, observe that, by using (6.2) and $E=E^{*}$ a.e., we have 
for $c>0$,

$$
\begin{aligned}
c^{-\kappa}(G(x, c(t+u))-G(x, c u)) \\
=G_{1}(x) c^{-\kappa}\left((c(t+u)+g(x))_{+}^{\kappa}-(c u+g(x))_{+}^{\kappa}\right) \\
\quad+G_{2}(x) c^{-\kappa}\left((c(t+u)+g(x))_{-}^{\kappa}-(c u+g(x))_{-}^{\kappa}\right) \\
=G\left(x, t+u+g_{c}(x)\right)-G\left(x, u+g_{c}(x)\right)
\end{aligned}
$$

a.e. $(x, u) \in E^{*} \times \mathbb{R}$, where $g_{c}(x)=\left(c^{-1}-1\right) g(x)$. Therefore, the process $X_{\alpha}^{(2)}$ is also generated by an identity flow on $E^{*}$ and a related semi-additive functional $\left\{g_{c}\right\}_{c>0}$. Now, if the representation $\{G(x, t+u)-G(x, u), x \in X, u \in \mathbb{R}\}_{t \in \mathbb{R}}$ is minimal for the process $X_{\alpha}$, then this representation restricted to $E^{*} \times \mathbb{R}$ is also minimal for the process $X_{\alpha}^{(2)}$. This fact is a consequence of the definition of a minimal representation [see Pipiras and Taqqu (2001a)] or can also be obtained by using Theorem 3.8 in Rosiński (1998). It follows by using the uniqueness of the flow in Theorem 4.1 of Pipiras and Taqqu (2001a) and also the relations (10.6) and (10.7) that for all $c>0, \psi_{c}^{*}(x)=x$ a.e. for $x \in E^{*}$. We then deduce that $\psi_{c}(x)=x$ a.e. for $(x, c) \in E \times(0, \infty)$. Then, for a.e. $x \in E, \psi_{c}(x)=x$ a.e. $d c$. By using the flow property (2.5), we get that, for a.e. $x \in E, \psi_{c}(x)=x$ for all $c>0$. This shows that $E \subset F$ a.e., where $F$ denotes the set on the right-hand side of (10.5). The inclusion $F \subset E$ a.e. follows as in the proof of Theorem 5.1 by showing that the relation (5.3), valid for a.e. $(x, u) \in F \times \mathbb{R}$, implies the relation (5.7), valid for a.e. $(x, z) \in F \times \mathbb{R}$.

The following result follows directly from Theorem 10.1.

COROLlaRY 10.1. Under the assumptions of Theorem 10.1, we have

$$
X_{\alpha}^{(2)} \stackrel{d}{=} X_{\alpha}^{F}, \quad X_{\alpha}^{(3)} \stackrel{d}{=} X_{\alpha}^{C \backslash F},
$$

where the processes $X_{\alpha}^{(2)}$ and $X_{\alpha}^{(3)}$ are defined by (7.2) and (7.3), respectively, and the processes $X_{\alpha}^{F}$ and $X_{\alpha}^{C \backslash F}$ are defined by (10.2) and (10.3), respectively.

\section{APPENDIX}

Almost everywhere version of the Cauchy functional equation. Recall that a function $k: \mathbb{R} \mapsto \mathbb{R}$ satisfies the Cauchy functional equation if $k(s+t)=$ $k(s)+k(t)$ for all $s, t \in \mathbb{R}$. It is well-known [see, e.g., Section 1.1.3 in Bingham, Goldie and Teugels (1987)] that the only measurable solutions to the Cauchy functional equation are of the form $k(s)=k(1) s$. We shall say that a function $k$ satisfies the Cauchy functional equation a.e. if the above equation holds a.e. $(s, t)$. The following proposition, used in the proof of Theorem 5.1 above, concerns functions that satisfy the Cauchy functional equation a.e. It may be useful in other contexts as well. 
Proposition A.1. Suppose that $k: \mathbb{R} \mapsto \mathbb{R}$ is a measurable function such that:

(i) $k(s+t)=k(s)+k(t)$ a.e. $d s d t$;

(ii) a.e. $d s, k(s+r)=k(s)+k(r)$ for each $r \in \mathbb{Q}$;

(iii) a.e. $d s, k(r s)=r k(s)$ for each $r \in \mathbb{Q}$; and

(iv) $k(r)=r k(1)$ for each $r \in \mathbb{Q}$.

Then $k(s)=k(1) s$ a.e. $d s$.

The proof of the proposition is based on the following lemma which is an a.e. analogue of Lemma 1.1.6 in Bingham et al. (1987).

LEMMA A.1. If a measurable function $k$ satisfies the condition (i) of Proposition A.1, then $k$ is bounded a.e. on some neighborhood of the origin.

PROOF. The basic idea is to arrive at relation (A.1) below where $s$ is around the origin and where $t_{0}, a$ and $a^{\prime}$ are the points where the function $k$ is bounded. By taking $M$ large enough, we may suppose that $k(t) \leq M<\infty$ for $t \in A$ with a set $A$ of positive Lebesgue measure. By Corollary 1.1.3 of Bingham, Goldie and Teugels (1987), $A+A=\left\{a+a^{\prime}: a \in A, a^{\prime} \in A\right\}$ contains some interval $(t-\delta, t+\delta)$ with $\delta>0$. Choose now $t_{0} \in(t-\delta, t+\delta)$ such that $k\left(s+t_{0}\right)=k(s)+k\left(t_{0}\right)$ a.e. $d s$ and choose also $\delta_{0}>0$ such that $\left(t_{0}-\delta_{0}\right.$, $\left.t_{0}+\delta_{0}\right) \subset(t-\delta, t+\delta)$. The set $Z=\left\{\left(a, a^{\prime}, s\right): a+a^{\prime}=s+t_{0}, a, a^{\prime} \in A\right\}$ and its $\operatorname{proj}_{s} Z=\left\{s: \exists a, a^{\prime}\right.$ such that $\left.\left(a, a^{\prime}, s\right) \in Z\right\}$ are measurable, and $\left(-\delta_{0}, \delta_{0}\right) \subset$ $\operatorname{proj}_{s} Z$, that is, we can choose $s \in \operatorname{proj}_{s} Z$ around the origin. Now let $Z_{0}=$ $\left\{\left(a, a^{\prime}, s\right): k\left(a+a^{\prime}\right)=k(a)+k\left(a^{\prime}\right), s \in \mathbb{R}\right\}$. By (i), $Z_{0}=\mathbb{R}^{3}$ a.e. $d a d a^{\prime} d s$. Then $Z \cap Z_{0}=Z$ a.e., $\operatorname{proj}_{s}\left(Z \cap Z_{0}\right)=\operatorname{proj}_{s} Z$ a.e. $d s$ and $\left(-\delta_{0}, \delta_{0}\right) \subset \operatorname{proj}_{s}\left(Z \cap Z_{0}\right)$ a.e. $d s$. It follows that, for a.e. $|s|<\delta_{0}$, namely such that $s \in \operatorname{proj}_{s}\left(Z \cap Z_{0}\right)$ and $k\left(s+t_{0}\right)=k(s)+k\left(t_{0}\right)$,

$$
k(s)+k\left(t_{0}\right)=k\left(s+t_{0}\right)=k\left(a+a^{\prime}\right)=k(a)+k\left(a^{\prime}\right)
$$

and hence $k(s)=k(a)+k\left(a^{\prime}\right)-k\left(t_{0}\right) \leq 2 M-k\left(t_{0}\right)$. The bound from below will follow by similar arguments since, by (i), $k(s)=-k\left(s_{0}-s\right)+k\left(s_{0}\right)$ a.e. $d s$ for some fixed $s_{0} \in \mathbb{R}$.

Proof of Proposition A.1. By Lemma A.1, there are $M, \delta>0$ with $|k(s)| \leq M$ a.e. for $|s|<\delta$. Suppose without loss of generality that $\delta=1$. Then, by condition (iii), $|k(s)| \leq M / n$ a.e. for $0<s<1 / n$. It follows that $|k(s-[s n] / n)| \leq$ $M / n$ a.e. $d s$ for $s \in \mathbb{R}$. By condition (ii), $k(s-[s n] / n)=k(s)-k([s n] / n)$ a.e. $d s$ for $s \in \mathbb{R}$. Then, by (iv), for a.e. $s \in \mathbb{R}$,

$$
|k(s)-s k(1)|=|k(s-[s n] / n)+([s n] / n-s) k(1)| \leq(M+|k(1)|) / n .
$$

Letting $n \rightarrow \infty$, we obtain $k(s)=k(1) s$ a.e. $d s$. 
Acknowledgment. The authors would like to thank an anonymous referee for useful comments.

\title{
REFERENCES
}

Bingham, N. H., Goldie, C. M. and Teugels, J. L. (1987). Regular Variation. Cambridge Univ. Press.

BURNECKI, K., RosińSKI, J. and WERON, A. (1998). Spectral representation and structure of stable self-similar processes. In Stochastic Processes and Related Topics: In Memory of Stamatis Cambanis 1943-1995 (I. Karatzas, B. S. Rajput and M. S. Taqqu, eds) 1-14. Birkhäuser, Boston.

Cambanis, S., Maejima, M. and Samorodnitsky, G. (1992). Characterization of linear and harmonizable fractional stable motions. Stochastic Processes Appl. 42 91-110.

CHI, Z. (2001). Construction of stationary self-similar generalized fields by random wavelet expansion. Probab. Theory Related Fields 121 269-300.

Kasahara, Y., Maejima, M. and VervaAt, W. (1988). Log-fractional stable processes. Stochastic Processes Appl. 30 329-339.

Krengel, U. (1969). Darstellungssätze für Strömungen und Halbströmungen II. Math. Ann. 182 $1-39$.

Krengel, U. (1985). Ergodic Theorems. De Gruyter, Berlin.

PIPIRAS, V. and TAQQU, M. S. (2000). The limit of a renewal-reward process with heavy-tailed rewards is not a linear fractional stable motion. Bernoulli 6 607-614.

Pipiras, V. and TAQQU, M. S. (2001a). Decomposition of self-similar stable mixed moving averages. Probab. Theory Related Fields. To appear.

PIPIRAS, V. and TAQQU, M. S. (2001b). Dilated fractional stable motions. Preprint.

Rosińs KI, J. (1995). On the structure of stationary stable processes. Ann. Probab. 23 1163-1187.

RosińSKI, J. (1998). Minimal integral representations of stable processes. Preprint.

SAMORODNITSKY, G. and TAQQU, M. S. (1990). (1/ $\alpha)$-self-similar processes with stationary increments. J. Multivariate Anal. 35 308-313.

Samorodnitsky, G. and Taqqu, M. S. (1994). Stable Non-Gaussian Processes: Stochastic Models with Infinite Variance. Chapman and Hall, New York.

Surgailis, D., Rosiński, J., Mandrekar, V. and Cambanis, S. (1993). Stable generalized moving averages. Probab. Theory Related Fields 97 543-558.

TAKENAKA, S. (1991). Integral-geometric construction of self-similar stable processes. Nagoya Math. J. 123 1-12.

\author{
DEPARTMENT OF MATHEMATICS \\ BOSTON UNIVERSITY \\ 111 CUMMINGTON STREET \\ BOSTON, MASSACHUSETTS 02215 \\ E-MAIL: pipiras@math.bu.edu \\ murad@math.bu.edu
}

